Supporting Information for:

\title{
Determination of the mechanical properties of model lipid bilayers using atomic force microscopy indentation
}

\author{
Dominik Drabik, ${ }^{\mathrm{a}}$ Martynas Gavutis, ${ }^{\mathrm{b}}$ Ramūnas Valiokas, ${ }^{\mathrm{b}}$ and Artūras Ulčinas ${ }^{\mathrm{b} *}$ \\ ${ }^{a}$ Laboratory of Cytobiochemistry, Faculty of Biotechnology, University of Wrocław, F. Joliot-Curie 14a, 50-383 Wrocław, Poland. \\ ${ }^{b}$ Department of Nanoengineering, Center for Physical Sciences and Technology, Savanorių 231, LT-02300 Vilnius, Lithuania \\ * Corresponding author : ulcinas@ftmc.lt
}

\begin{abstract}
Number of:
pages - 24

figures - 22

tables - 3
\end{abstract}




\section{Surface anchors and filler molecules}

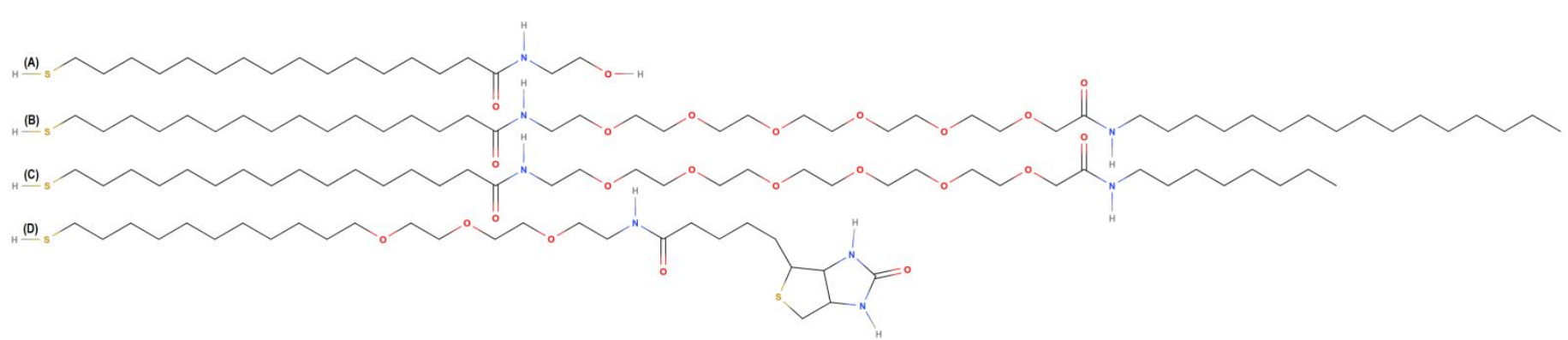

Figure S1. Chemical structure of surface anchors and filler molecules used for C8, C16 and STPV surface preparation. (A) filler molecule $\mathrm{HS}\left(\mathrm{CH}_{2}\right)_{15} \mathrm{CONHCH}_{2} \mathrm{CH}_{2} \mathrm{OH}$ (B) $\mathrm{C} 8$ anchor molecule $\mathrm{HS}\left(\mathrm{CH}_{2}\right)_{15} \mathrm{CONH}-\left(\mathrm{CH}_{2} \mathrm{CH}_{2} \mathrm{O}\right)_{6} \mathrm{CH}_{2} \mathrm{CONH}-(\mathrm{CD} 2)_{7} \mathrm{CD}{ }_{3}(\mathrm{C}) \mathrm{C} 16$ anchor molecule $\mathrm{HS}\left(\mathrm{CH}_{2}\right)_{15} \mathrm{CONH}-\left(\mathrm{CH}_{2} \mathrm{CH}_{2} \mathrm{O}\right)_{6} \mathrm{CH}_{2} \mathrm{CONH}-\left(\mathrm{CH}_{2}\right)_{15} \mathrm{CH}_{3}(\mathrm{D})$ biotin molecule $\mathrm{HS}\left(\mathrm{CH}_{2}\right)_{11}\left(\mathrm{CH}_{2} \mathrm{CH}_{2} \mathrm{O}\right)_{3}$ biotinyl.

\section{Colloidal probe preparation}

For measurements using a spherical indenter, silica colloidal particles with nominal $6.65 \mu \mathrm{m}$ diameter (microParticles, Germany) were attached to the tipless silicon nitride probes with nominal $0.1 \mathrm{~N} \cdot \mathrm{m}-1$ spring constant (NuNano, UK) using the UV-curable glue (NOA68, Norland), taking care to control the amount of glue on the cantilever. The attachment of the microsphere and its diameter was inspected by optical microscopy (x50 lens, BX51, Olympus), yielding the expected diameter of $7.1 \pm 0.36 \mu \mathrm{m}$ (specifically 6.65 , 7.5, 6.67 and $7.1 \mu \mathrm{m}$ depending on the probe used). Before each indentation experiment, the spring constant of the probe was calibrated in air using contact-based spring constant method. Briefly, the sensitivity of the deflection detection system was determined from the force distance curve of a hard substrate. Following this, the tip was retracted from the surface and the spring constant was determined using the thermal noise (equipartition) method.

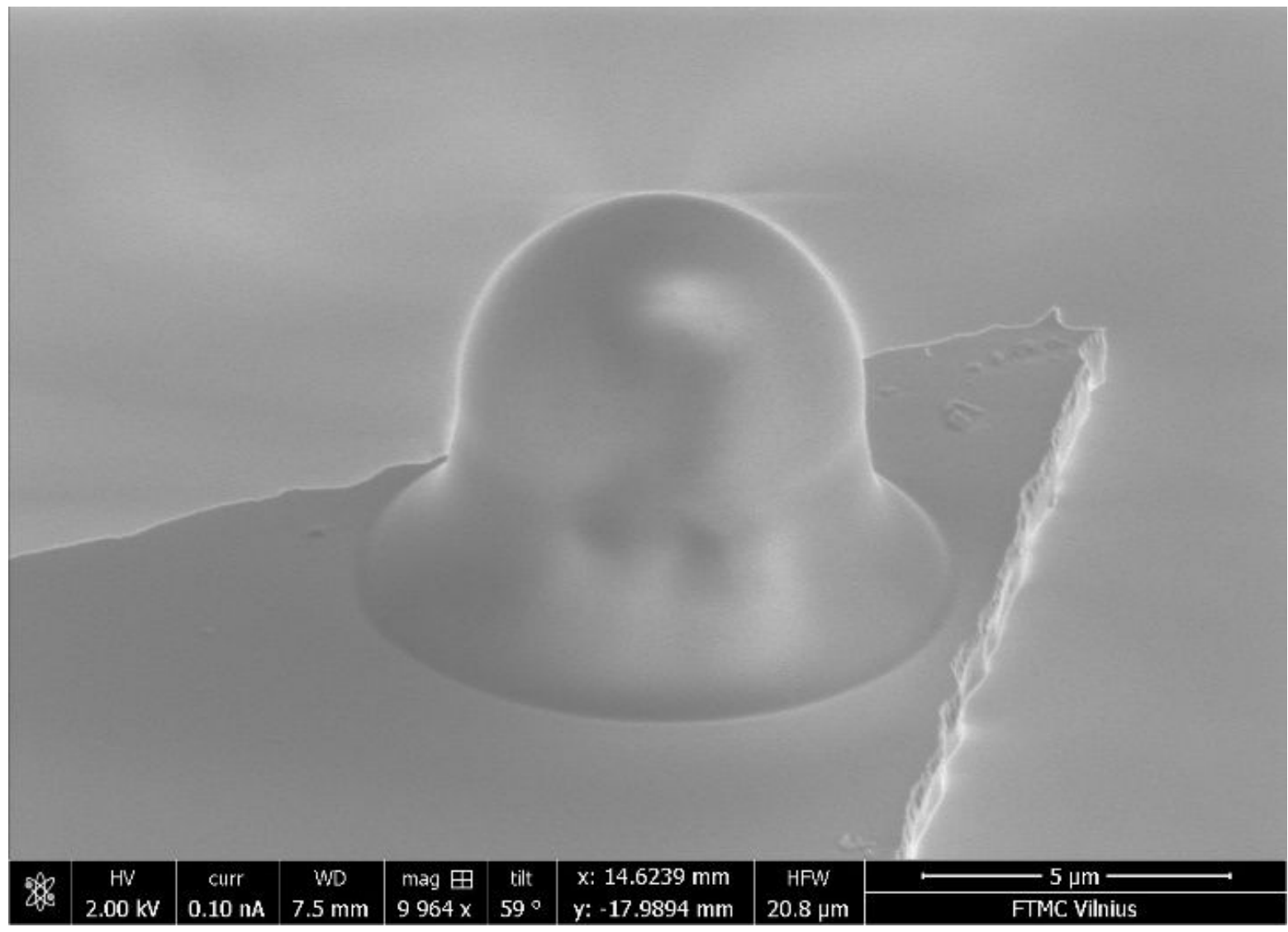

Figure S2. Scanning electron microscopy image of the colloidal particle (spherical) tip on the AFM cantilever.

\section{Model Assessment}

In this section we presented individual repeats for each of the measurements carried out on different samples, with different models and with two of the investigated tips: the cone-shaped and microsphere. 

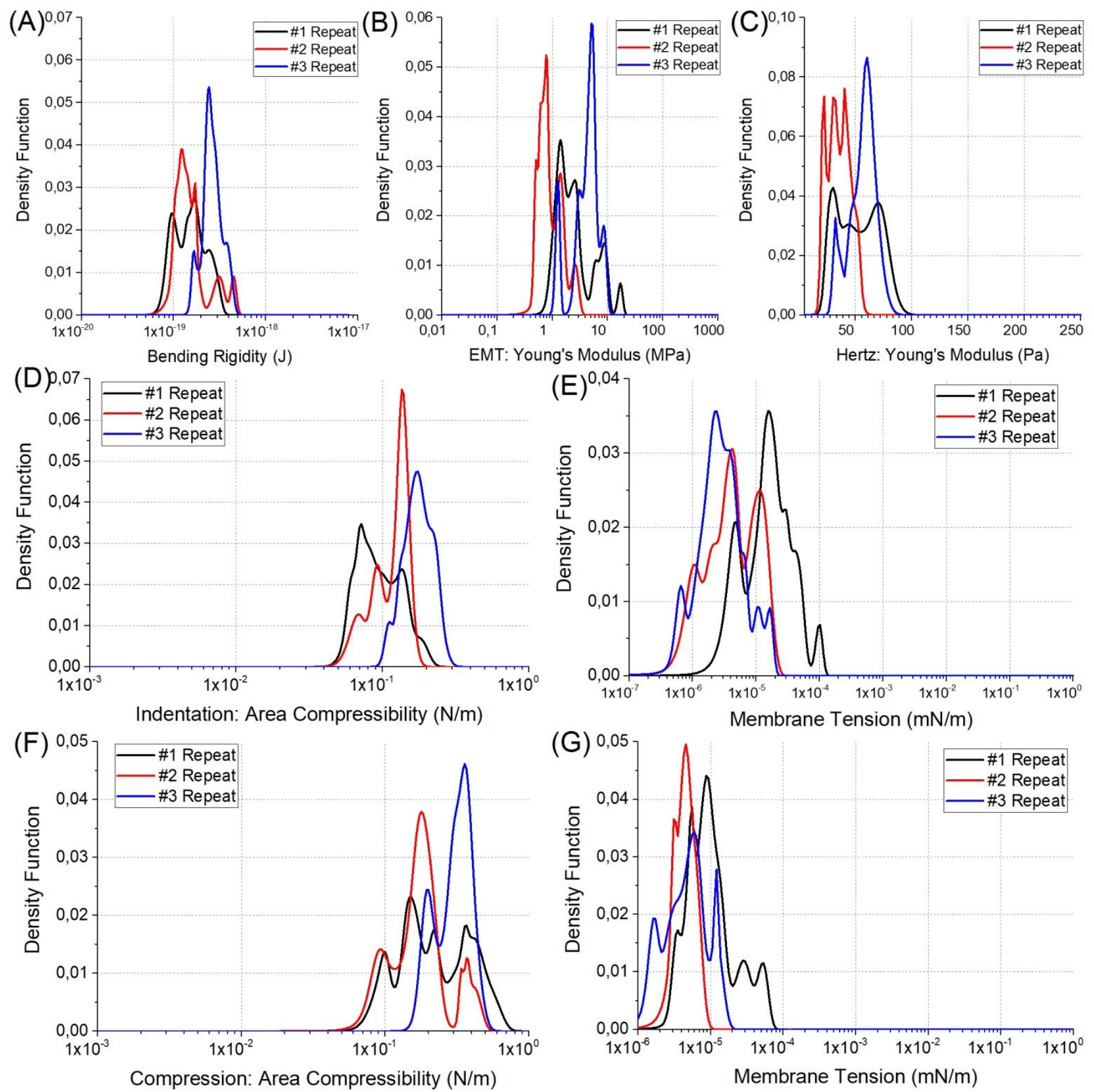

Figure S3. Kernel density representation for parameters obtained from model fitting for of each of the repeats. The results were obtained using silicon as a substrate and a cone-shaped tip. (A) bending rigidity obtained from the Reissner model. (B) Young's modulus from the Elastic Membrane Theory. (C) Young's modulus from the Hertz model. (D-E) area compressibility $\mathrm{K}_{\mathrm{A}}$ and membrane tension $\sigma$ for indentation model are shown, respectively. (F-G) area compressibility $\mathrm{K}_{\mathrm{A}}$ and membrane tension $\sigma$ for compression model, respectively. 

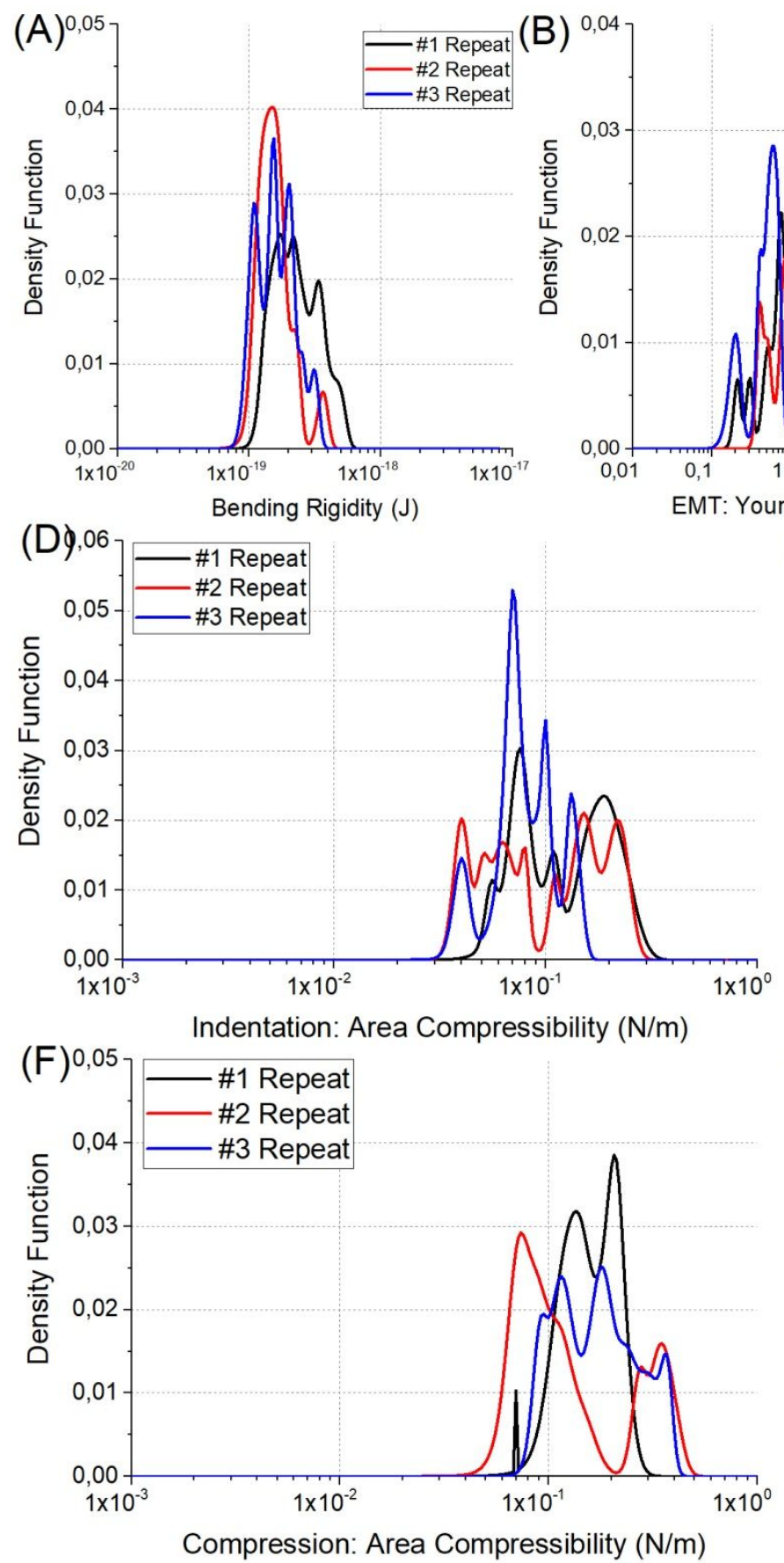
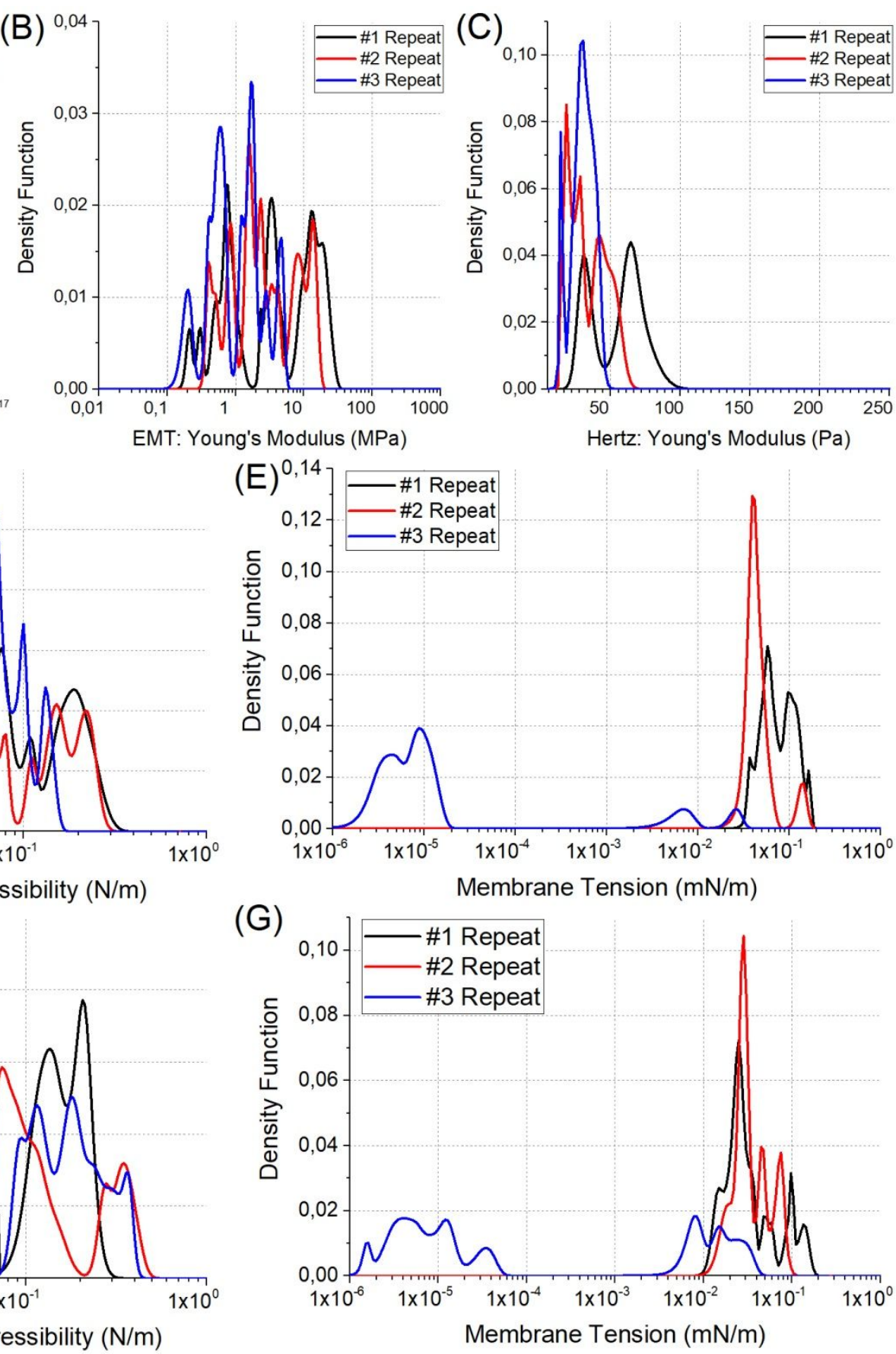

Figure S4. Kernel density representation for parameters obtained from model fitting for of each of the repeats. The results were obtained using the $\mathbf{C 8}$ anchor surface and cone-shaped tip. (A) bending rigidity obtained from the Reissner model. (B) Young's modulus from the Elastic Membrane Theory. (C) Young's modulus from the Hertz model. (D-E) area compressibility $\mathrm{K}_{\mathrm{A}}$ and membrane tension $\sigma$ for the indentation model, respectively. (F-G) area compressibility $\mathrm{K}_{\mathrm{A}}$ and membrane tension $\sigma$ for the compression model, respectively. 

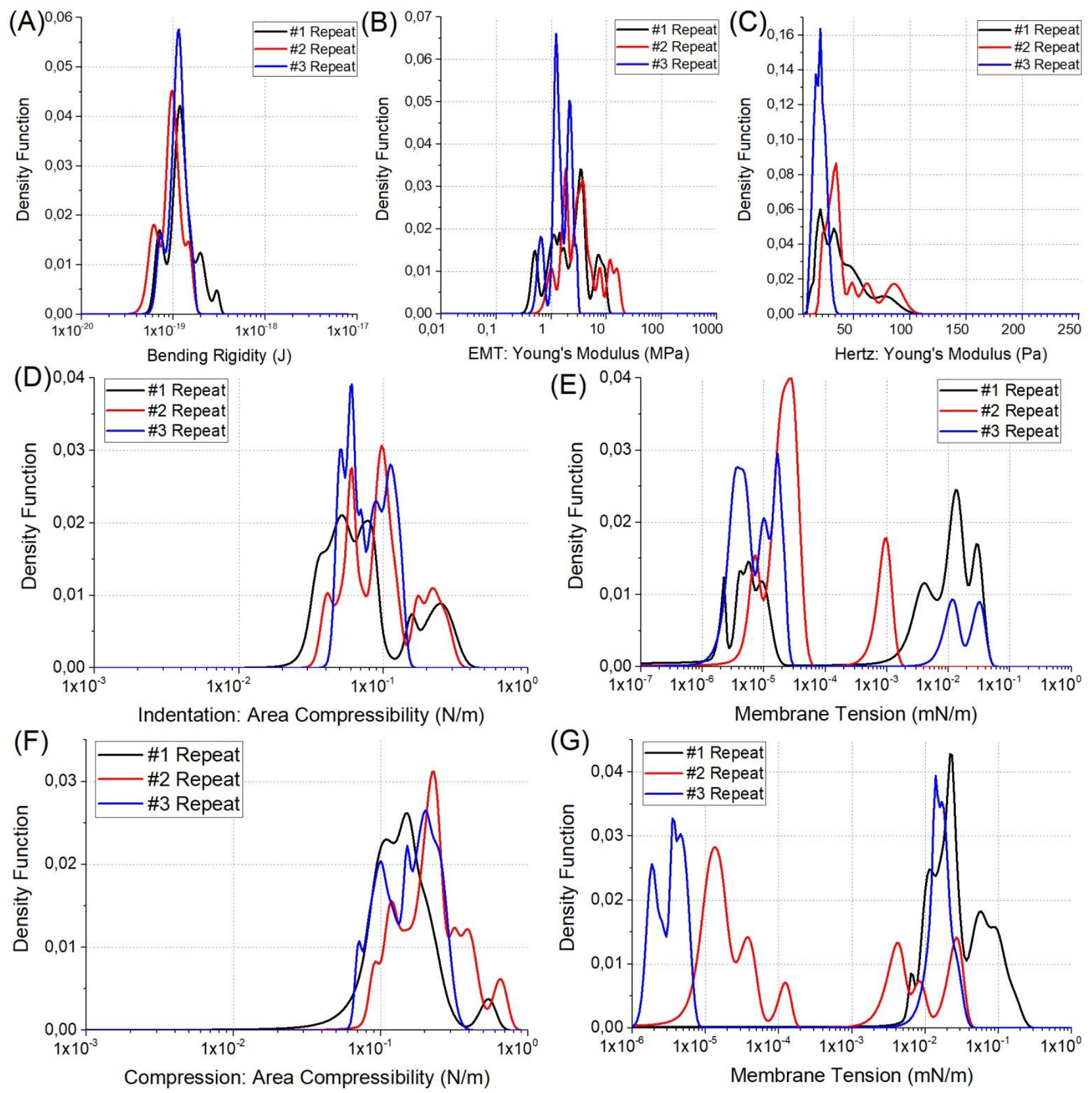

Figure S5. Kernel density representation for the parameters obtained from model fitting with distinguish for of each of the repeats. The results were obtained using the $\mathbf{C 1 6}$ anchor surface with the cone-shaped tip. (A) bending rigidity obtained from the Reissner model. (B) Young's modulus from the Elastic Membrane Theory. (C) Young's modulus from the Hertz model. (D-E) area compressibility $\mathrm{K}_{\mathrm{A}}$ and membrane tension $\sigma$ for the indentation model, respectively. (F-G) area compressibility $\mathrm{K}_{\mathrm{A}}$ and membrane tension $\sigma$ for the compression model, respectively. 

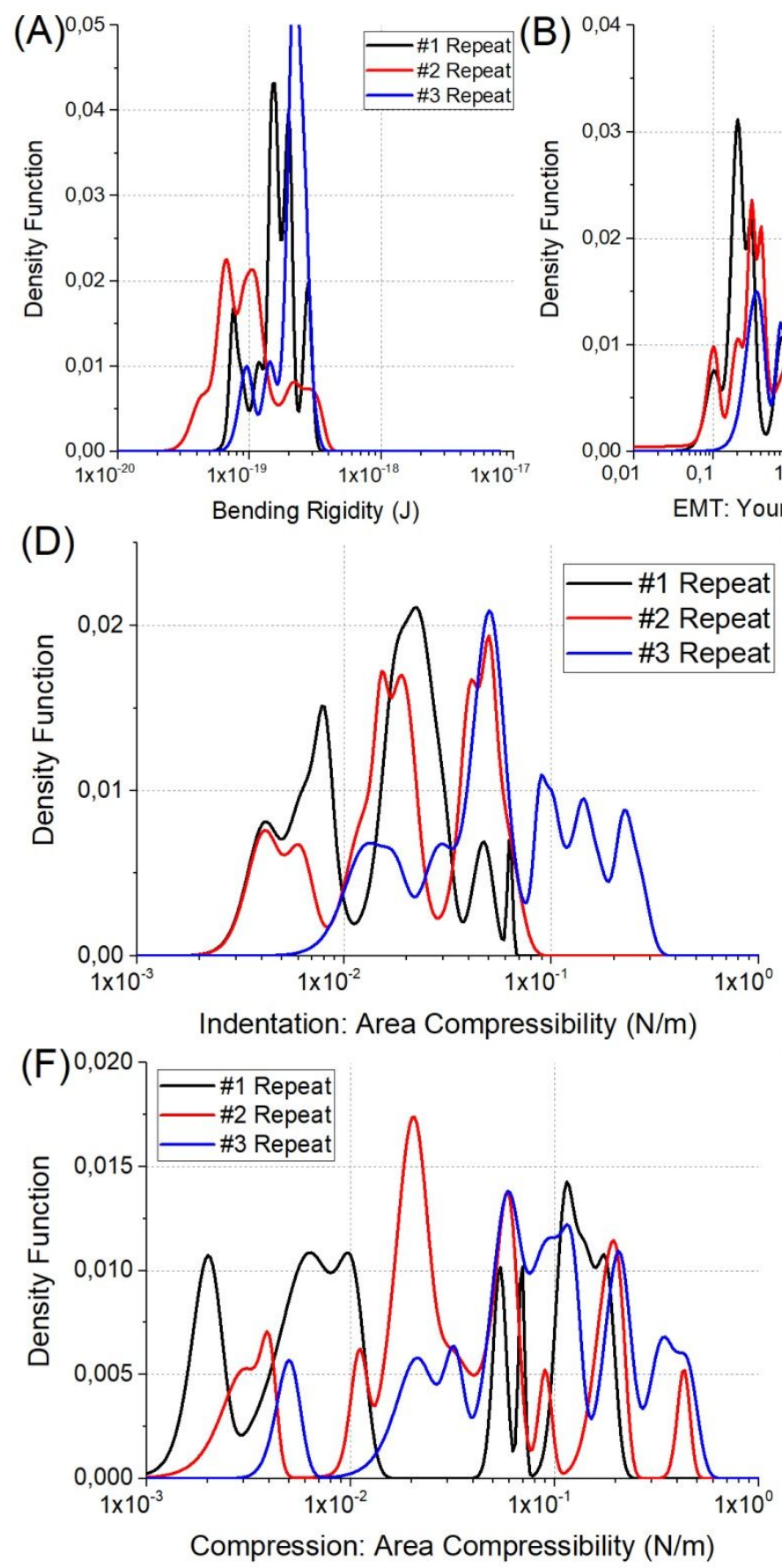
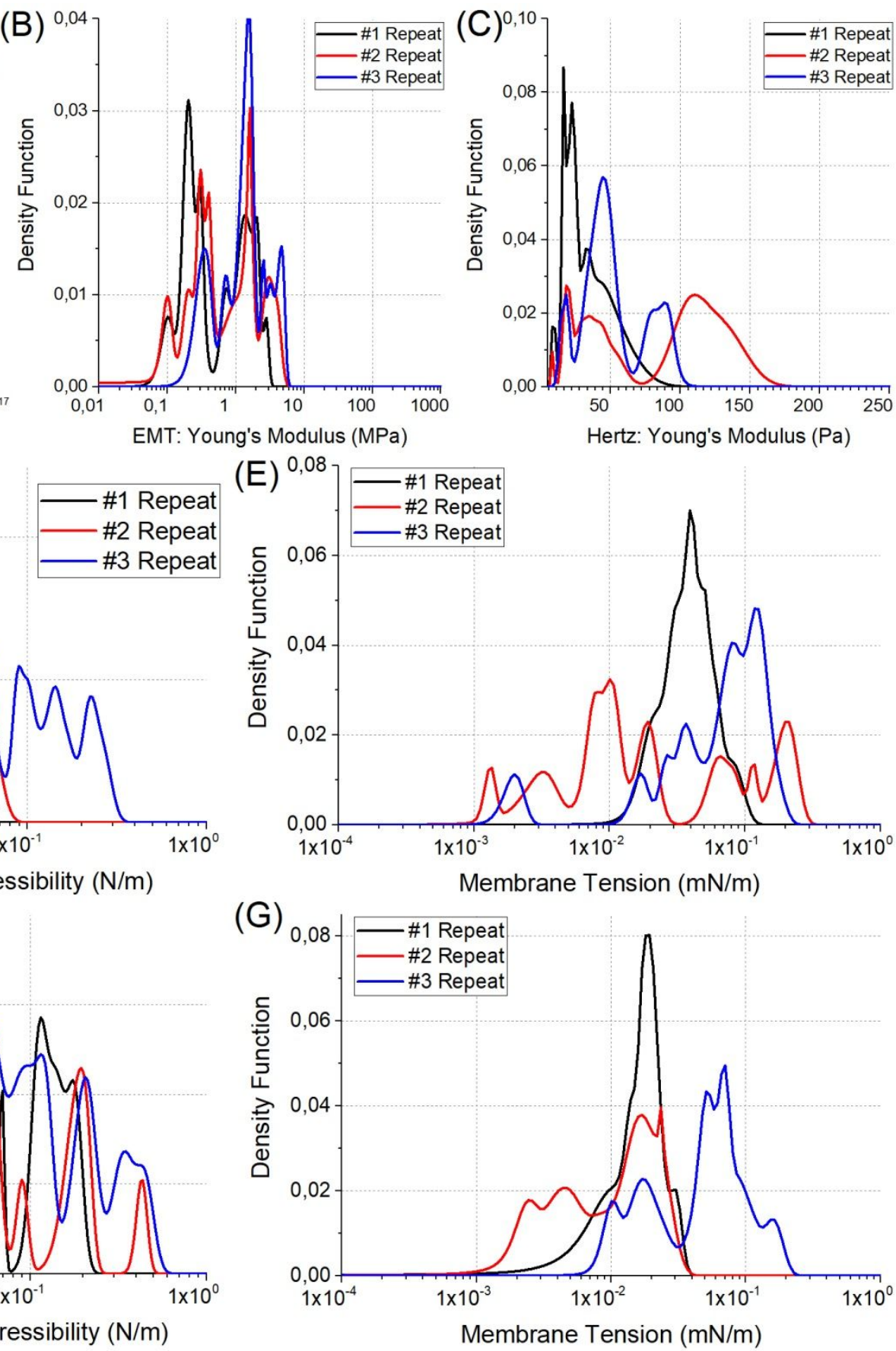

Figure S6. Kernel density representation for the parameters obtained from model fitting for of each of the repeats. The results were obtained using the STPV surface and cone-shaped tip. (A) bending rigidity obtained from the Reissner model. (B) Young's modulus from the Elastic Membrane Theory. (C) Young's modulus from the Hertz model. (D-E) area compressibility $\mathrm{K}_{\mathrm{A}}$ and membrane tension $\sigma$ for the indentation model, respectively. (F-G) area compressibility $\mathrm{K}_{\mathrm{A}}$ and membrane tension $\sigma$ for the compression model, respectively. 

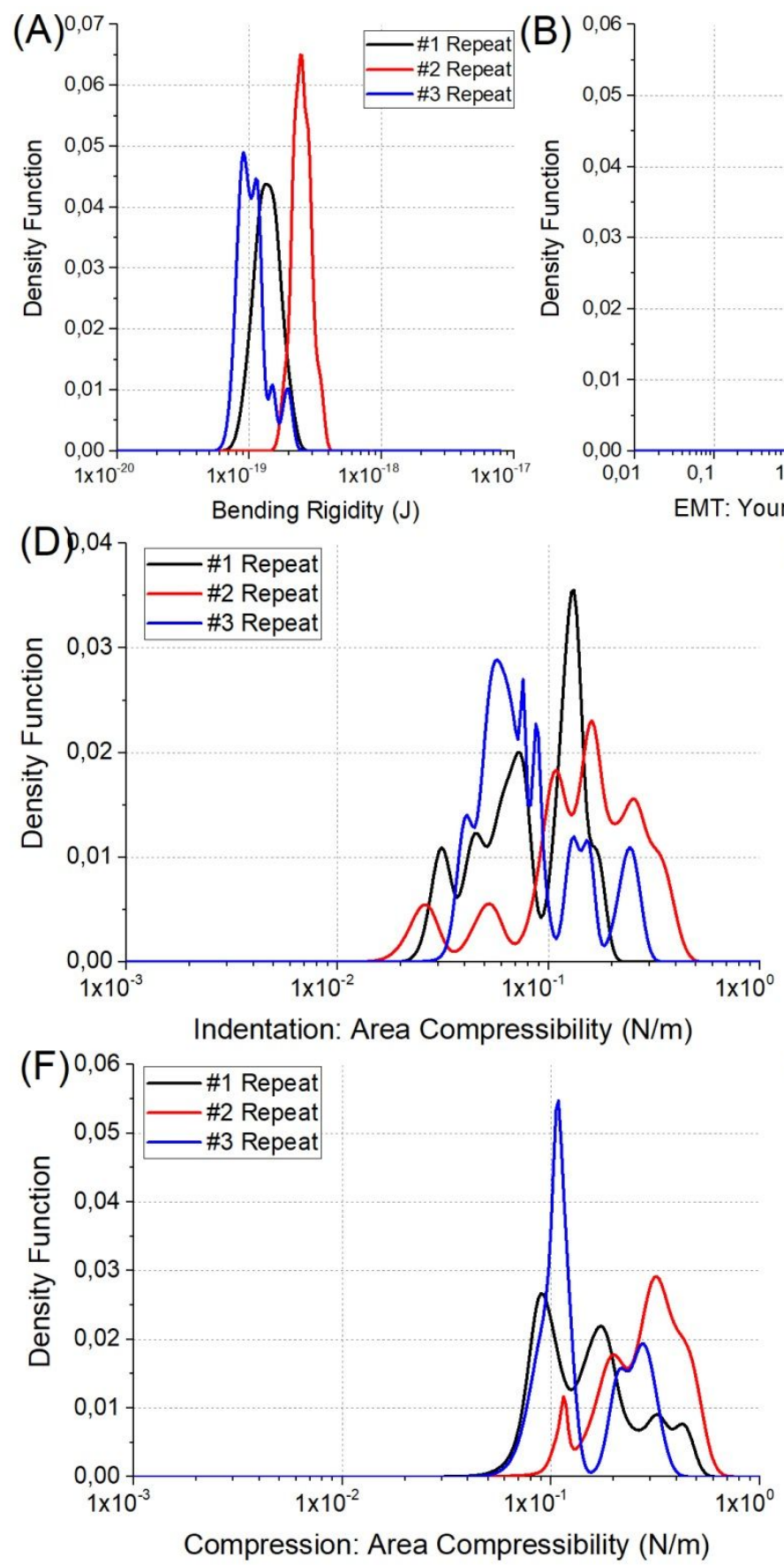
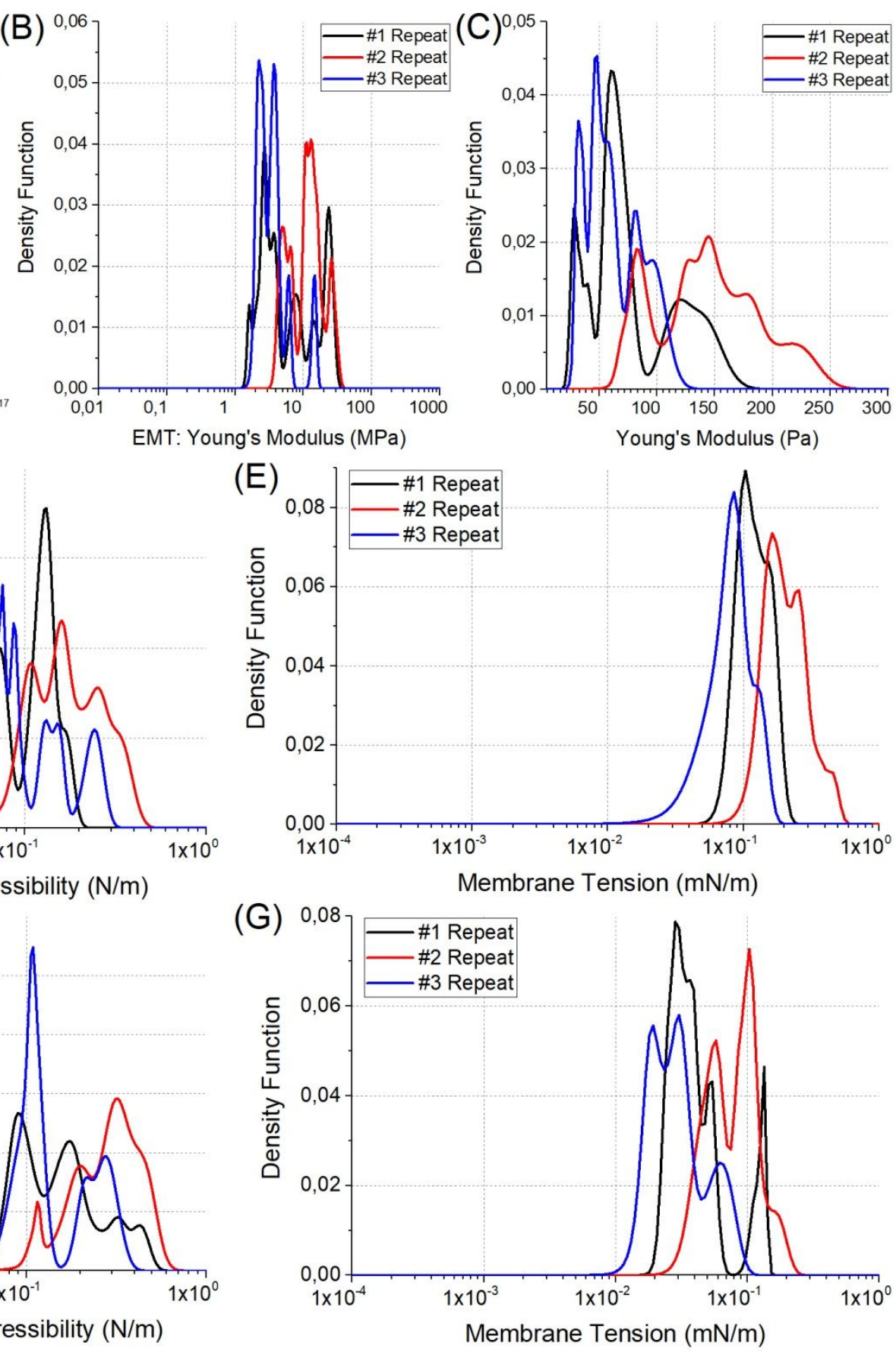

Figure S7. Kernel density representation for the parameters obtained from model fitting for of each of the repeats. The results were obtained using the silicon surface and sphere probe. (A) bending rigidity obtained from the Reissner model. (B) Young's modulus from the Elastic Membrane Theory. (C) Young's modulus from the Hertz model. (D-E) area compressibility $\mathrm{K}_{\mathrm{A}}$ and membrane tension $\sigma$ for the indentation model, respectively. (F-G) area compressibility $\mathrm{K}_{\mathrm{A}}$ and membrane tension $\sigma$ for the compression model, respectively. 

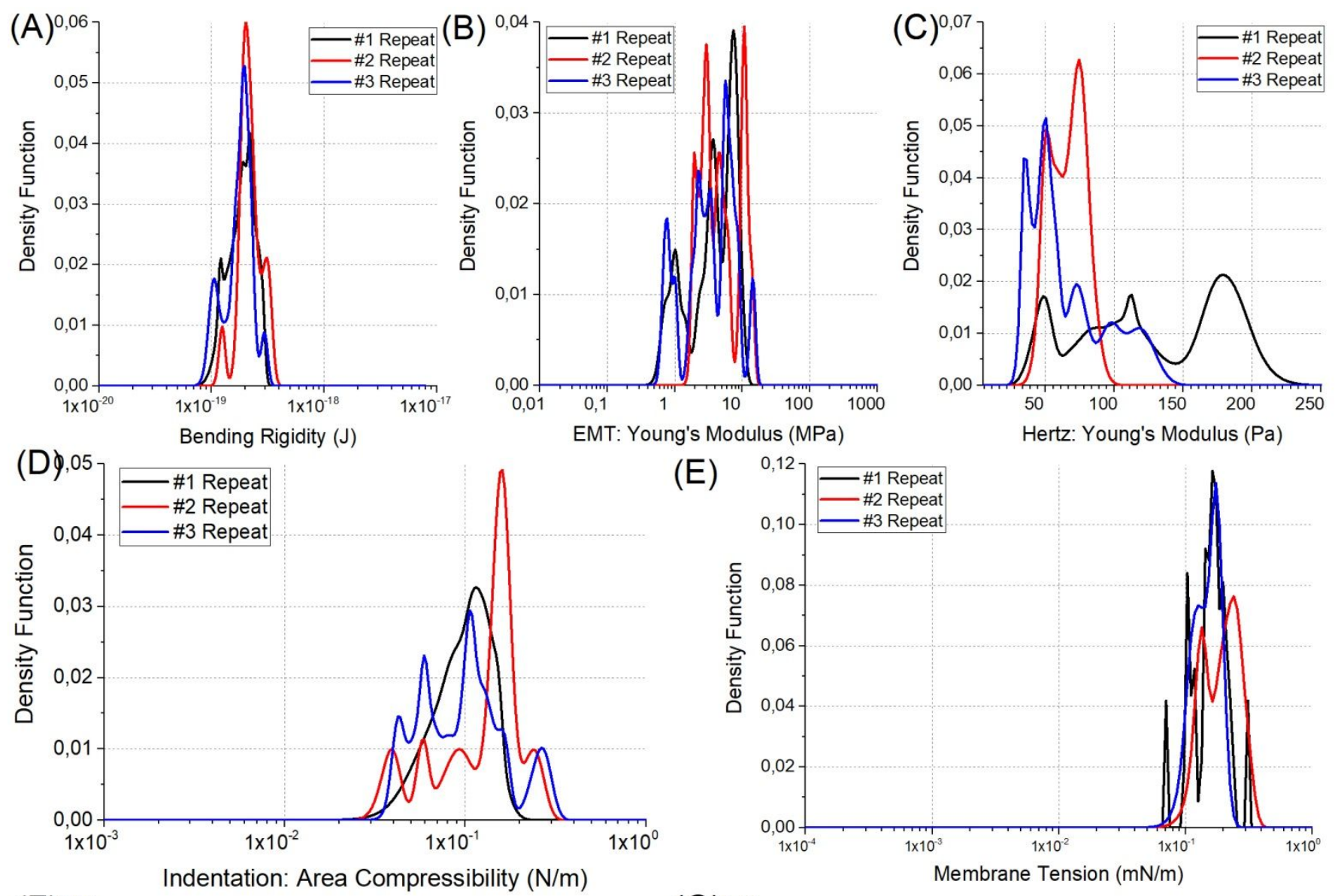

(E)
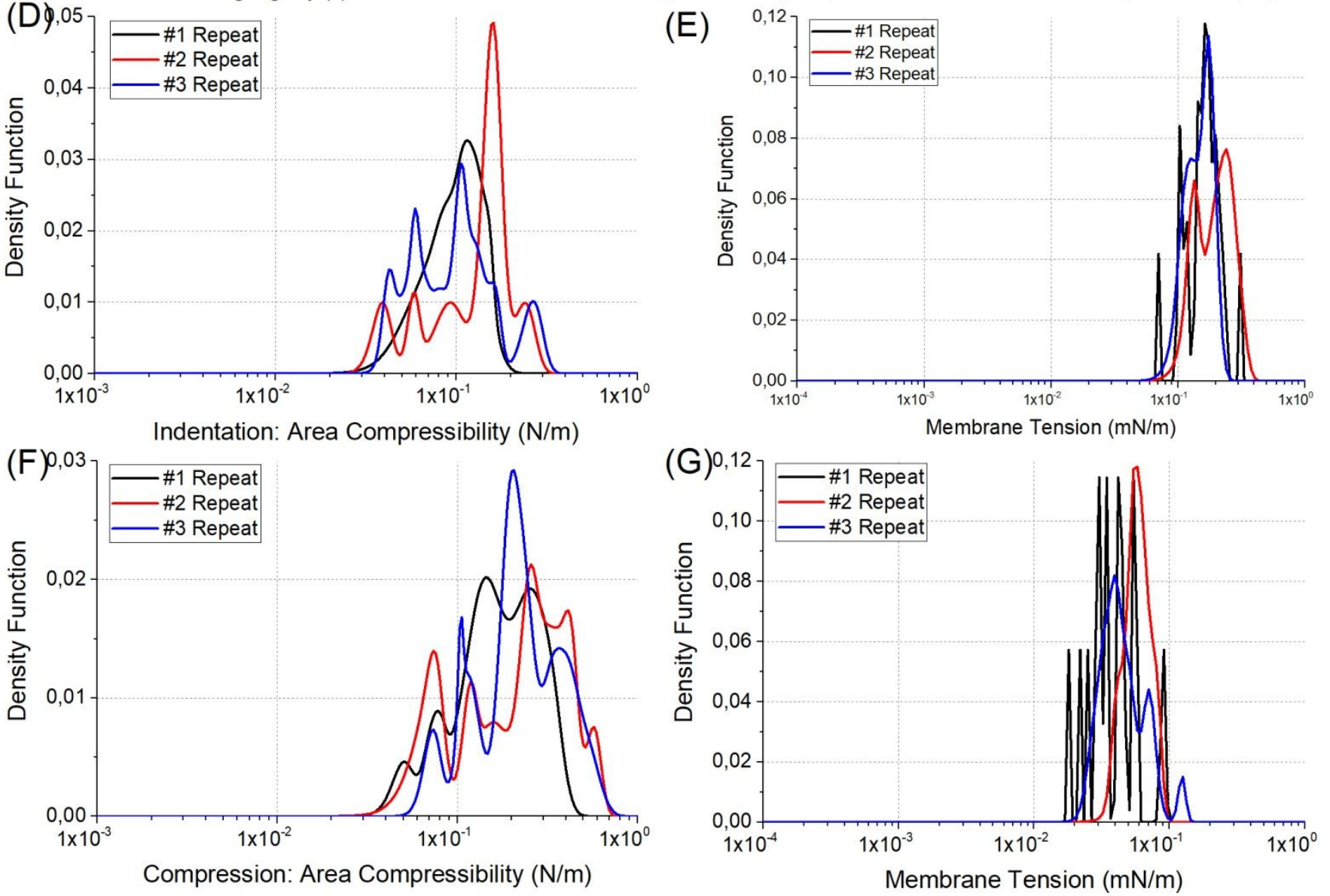

Figure S8. Kernel density representation for parameters obtained from model fitting for of each of the repeats. The results were obtained using the C8 anchor surface and sphere probe. (A) bending rigidity obtained from the Reissner model. (B) Young's modulus from the Elastic Membrane Theory. (C) Young's modulus from the Hertz model. (D-E) area compressibility $\mathrm{K}_{\mathrm{A}}$ and membrane tension $\sigma$ for the indentation model, respectively. (F-G) area compressibility $\mathrm{K}_{\mathrm{A}}$ and membrane tension $\sigma$ for the compression model, respectively.. 

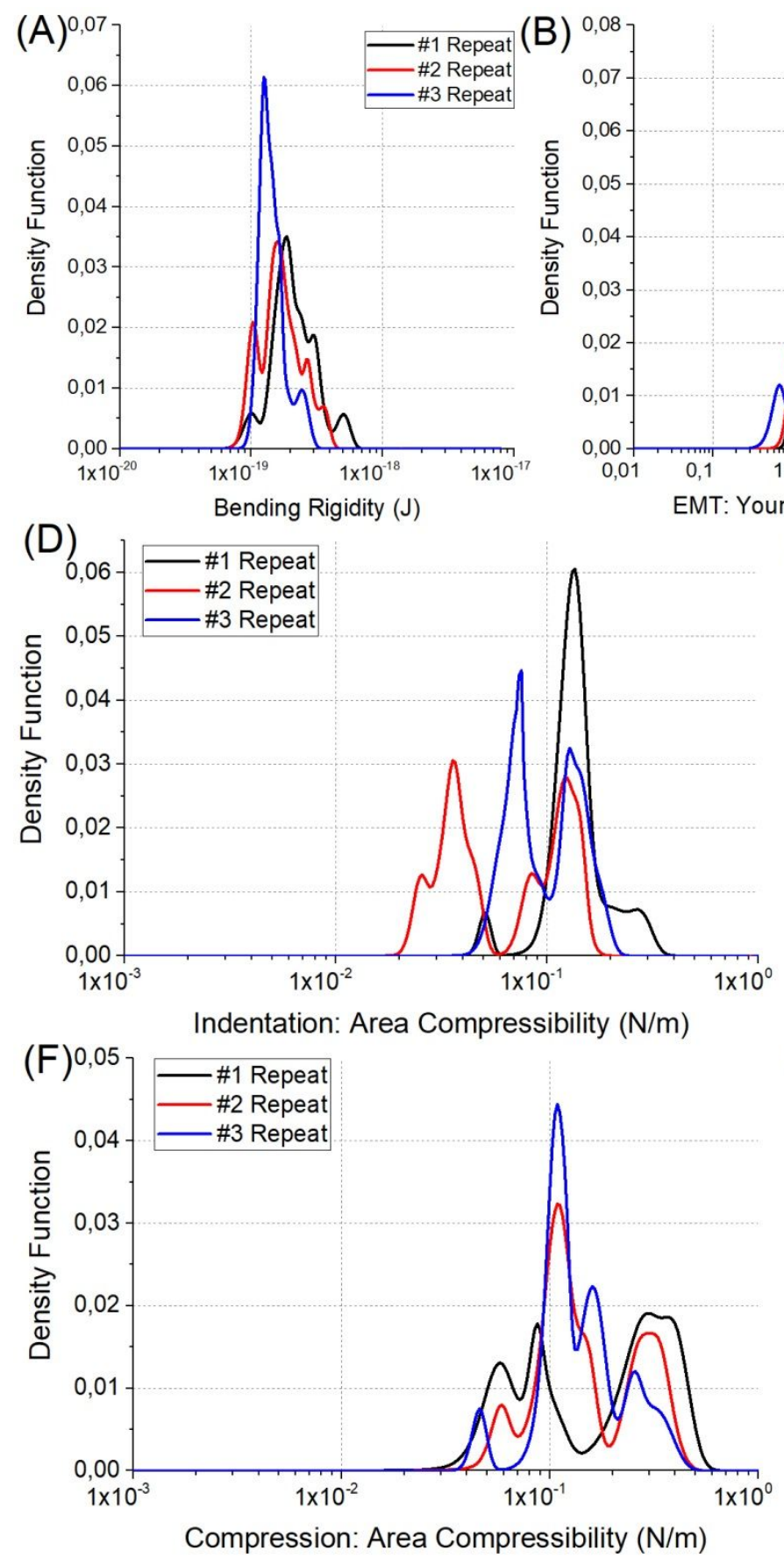
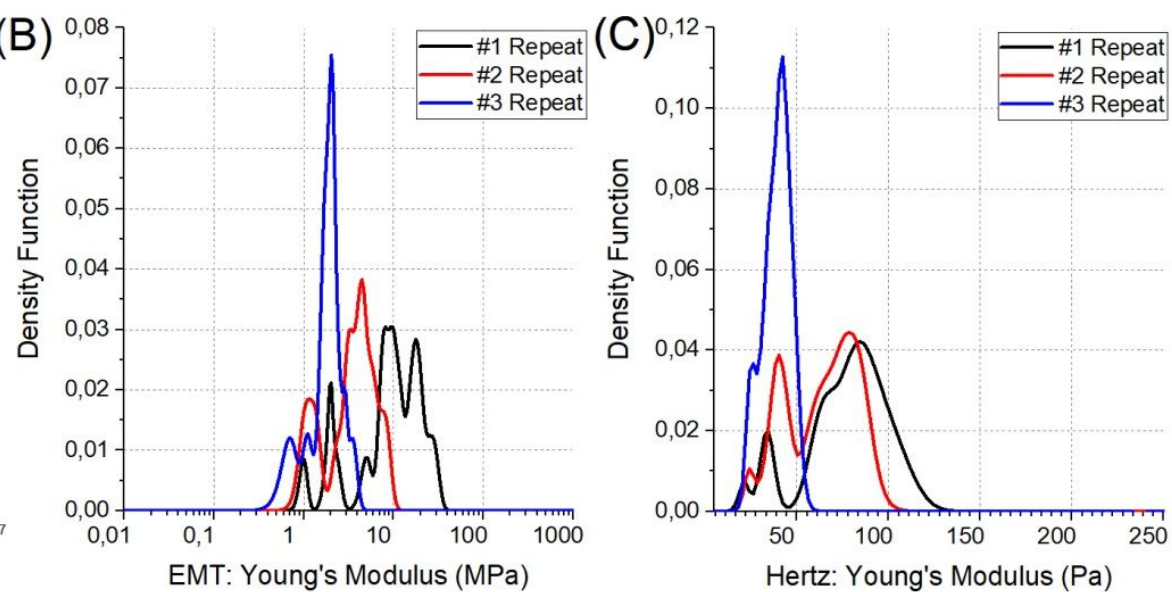

(E) $\left.{ }^{0,12}\right]$ \#1 Repeat
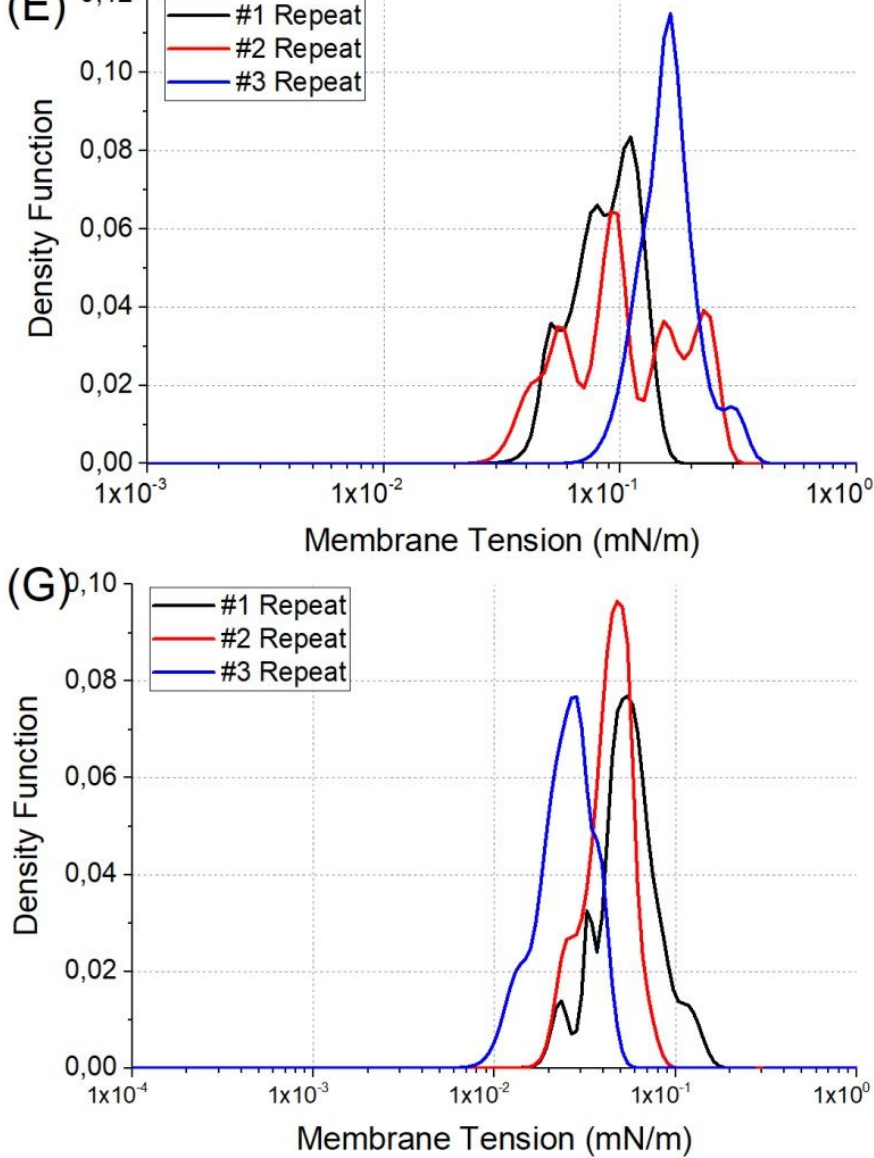

Figure S9. Kernel density representation for the parameters obtained from model fitting for of each of the repeats. The results were obtained using the $\mathbf{C 1 6}$ anchor surface and sphere probe. (A) bending rigidity obtained from the Reissner model. (B) Young's modulus from the Elastic Membrane Theory. (C) Young's modulus from the Hertz model. (D-E) area compressibility $\mathrm{K}_{\mathrm{A}}$ and membrane tension $\sigma$ for the indentation model, respectively. (F-G) area compressibility $\mathrm{K}_{\mathrm{A}}$ and membrane tension $\sigma$ for the compression model, respectively. 

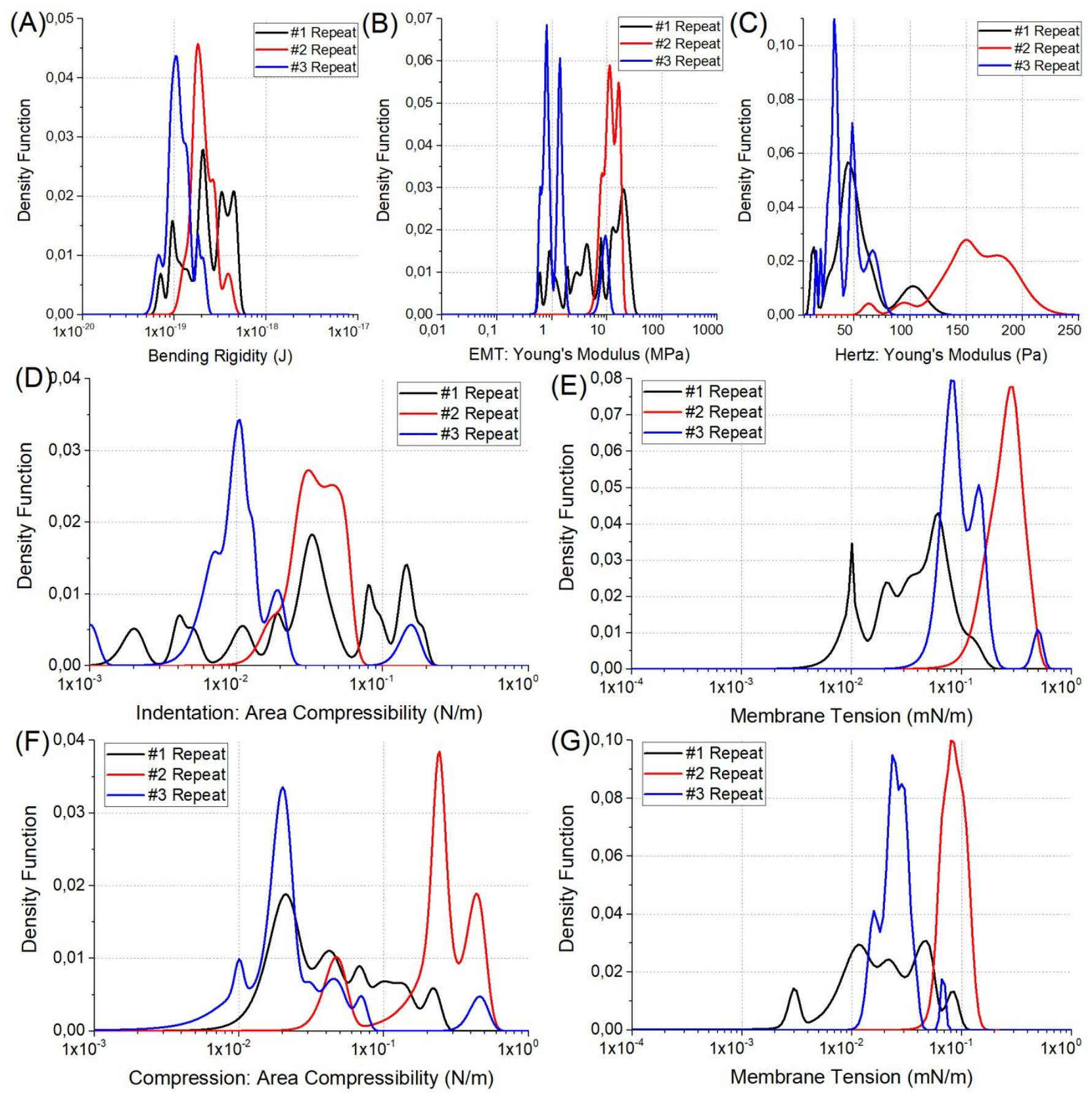

Figure S10. Kernel density representation for the parameters obtained from model fitting for of each of the repeats. The results were obtained using the STPV surface and sphere probe. (A) bending rigidity obtained from the Reissner model. (B) Young's modulus from the Elastic Membrane Theory. (C) Young's modulus from the Hertz model. (D-E) area compressibility $\mathrm{K}_{\mathrm{A}}$ and membrane tension $\sigma$ for the indentation model, respectively. (F-G) area compressibility $\mathrm{K}_{\mathrm{A}}$ and membrane tension $\sigma$ for the compression model, respectively.

\section{Surface Effect}

Below are presented the rest of the parameters for the measurements performed using the cone-shaped probe as well as the parameters for the measurements performed using the sphere probe. Also, a table with the parameters calculated as a median value \pm MAD (median absolute deviation) was included. 
Table S1. Median of parameters obtained from AFM indentation measurements.

\begin{tabular}{|c|c|c|c|c|c|}
\hline \multirow{2}{*}{ Parameter } & \multirow{2}{*}{ Tip } & \multicolumn{4}{|c|}{ Surface } \\
\hline & & Silicon & C8 anchor & C16 anchor & STPV \\
\hline \multirow{2}{*}{$\begin{array}{l}\text { bending rigidity } \\
\qquad \kappa[\mathrm{J}]\end{array}$} & Cone & $(1.8 \pm 0.6) \cdot 10^{-19}$ & $(1.7 \pm 0.4) \cdot 10^{-19}$ & $(1.1 \pm 0.2) \cdot 10^{-19}$ & $(1.5 \pm 0.6) \cdot 10^{-19}$ \\
\hline & Sphere & $(1.5 \pm 0.5) \cdot 10^{-19}$ & $(2.0 \pm 0.3) \cdot 10^{-19}$ & $(1.7 \pm 0.4) \cdot 10^{-19}$ & $(1.8 \pm 0.6) \cdot 10^{-19}$ \\
\hline \multirow{2}{*}{$\begin{array}{l}\text { area compressibility } \\
\mathrm{K}_{\mathrm{A}}[\mathrm{N} / \mathrm{m}] \underline{\mathrm{IND}}\end{array}$} & Cone & $0.13 \pm 0.04$ & $0.09 \pm 0.04$ & $0.08 \pm 0.03$ & $0.03 \pm 0.02$ \\
\hline & Sphere & $0.11 \pm 0.05$ & $0.11 \pm 0.04$ & $0.12 \pm 0.03$ & $0.03 \pm 0.02$ \\
\hline \multirow[t]{2}{*}{$\begin{array}{c}\text { membrane tension } \\
\sigma[\mathrm{mN} / \mathrm{m}] \underline{\mathrm{IND}}\end{array}$} & Cone & $(8 \pm 6)^{-} \cdot 10^{-6}$ & $\begin{array}{l}0.05 \pm 0.01 \\
(8 \pm 3) \cdot 10^{-6}\end{array}$ & $\begin{array}{l}0.008 \pm 0.004 \\
(9 \pm 6) \cdot 10^{-6}\end{array}$ & $0.04 \pm 0.03$ \\
\hline & Sphere & $0.17 \pm 0.04$ & $0.17 \pm 0.04$ & $0.12 \pm 0.06$ & $0.09 \pm 0.06$ \\
\hline \multirow{2}{*}{$\begin{array}{c}\text { area compressibility } \\
\mathrm{K}_{\mathrm{A}}[\mathrm{N} / \mathrm{m}] \underline{\mathrm{COM}}\end{array}$} & Cone & $0.22 \pm 0.08$ & $0.09 \pm 0.06$ & $0.16 \pm 0.08$ & $0.06 \pm 0.05$ \\
\hline & Sphere & $0.19 \pm 0.09$ & $0.2 \pm 0.1$ & $0.12 \pm 0.04$ & $0.043 \pm 0.023$ \\
\hline \multirow{2}{*}{$\begin{array}{c}\text { membrane tension } \\
\sigma[\mathrm{mN} / \mathrm{m}] \underline{\mathrm{COM}}\end{array}$} & Cone & $(6 \pm 3) \cdot 10^{-6}$ & $\begin{array}{l}0.03 \pm 0.02 \\
(9 \pm 6) \cdot 10^{-6}\end{array}$ & $\begin{array}{l}0.019 \pm 0.07 \\
(8 \pm 5) \cdot 10^{-6}\end{array}$ & $0.02 \pm 0.01$ \\
\hline & Sphere & $0.052 \pm 0.024$ & $0.046 \pm 0.012$ & $0.042 \pm 0.013$ & $0.03 \pm 0.02$ \\
\hline \multirow{2}{*}{$\begin{array}{l}\text { Young's Modulus } \\
{[\mathrm{MPa}] \underline{\mathrm{EMT}}}\end{array}$} & Cone & $2.0 \pm 1.2$ & $1.8 \pm 1.3$ & $2.1 \pm 0.9$ & $1.1 \pm 0.8$ \\
\hline & Sphere & $6 \pm 4$ & $4.4 \pm 2.3$ & $3.1 \pm 1.5$ & $8 \pm 7$ \\
\hline \multirow{2}{*}{$\begin{array}{l}\text { Young's Modulus } \\
{[\mathrm{Pa}] \underline{\text { Hertz }}}\end{array}$} & Cone & $41 \pm 12$ & $28 \pm 10$ & $24 \pm 8$ & $27 \pm 11$ \\
\hline & Sphere & $70 \pm 30$ & $66 \pm 18$ & $45 \pm 19$ & $49 \pm 22$ \\
\hline
\end{tabular}

Presented values were medians over all 3 repeats performed on 3 different substrate samples. In case of clear presence of two populations (mainly membrane tensions for the C8 and C16 anchor surfaces) a value was presented for each of the populations. It should be noted that the experimental data did not follow normal distributions, therefore median values were presented instead of averages. Abbreviations used: IND - indentation model, COM - compression model, EMT - elastic membrane theory model. 

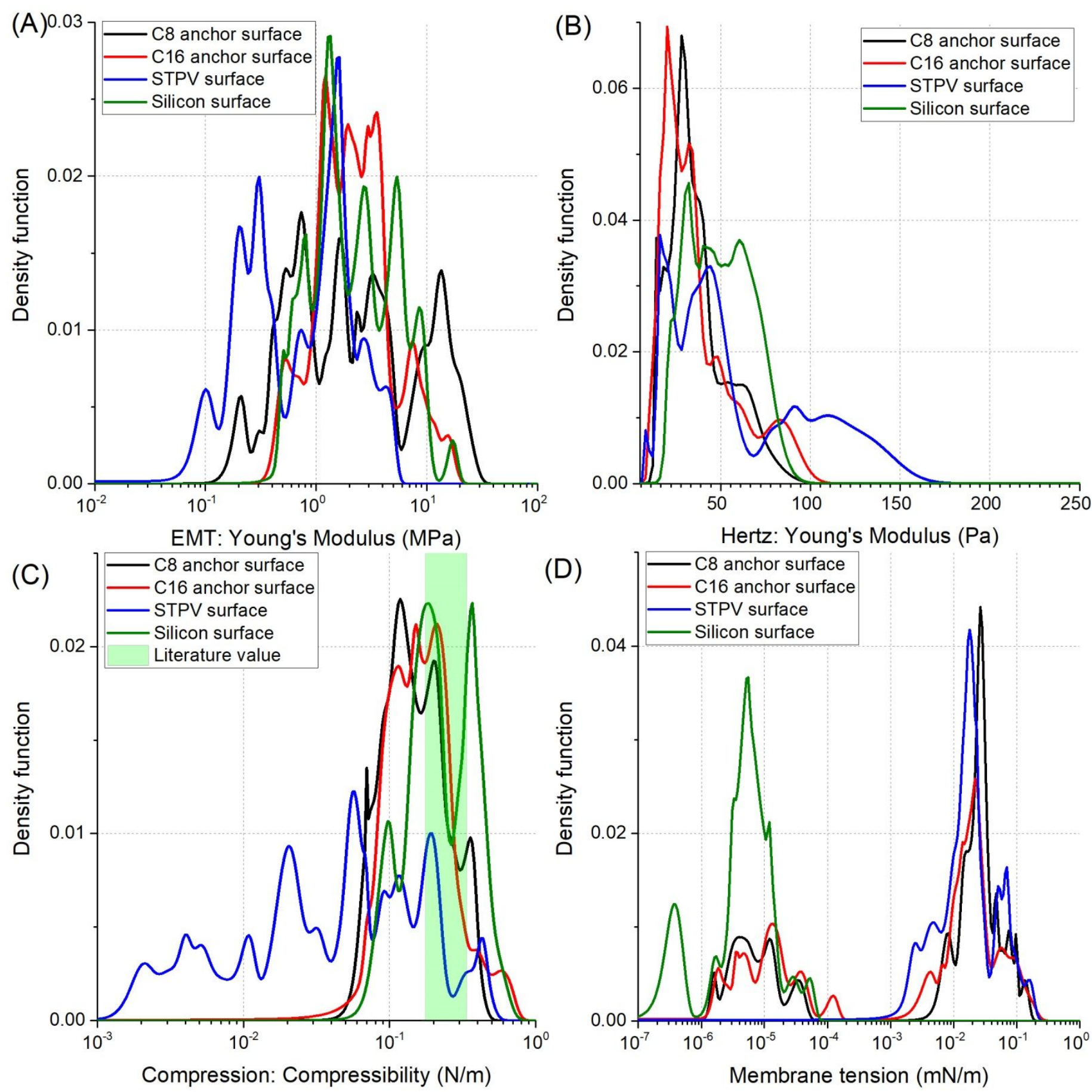

Figure S11. Kernel density representation for the parameters obtained from model fitting in function for the different substrates. The results were obtained from the measurements performed using the cone-shaped tip. (A) Young's modulus from the Elastic Membrane Theory. (B) Young's modulus from the Hertz model. (C-D) area compressibility $\mathrm{K}_{\mathrm{A}}$ and membrane tension $\sigma$ for the compression model, respectively.

\section{Statistical significance}

EMT: Young's modulus - significant difference with both statistical tests. Tukey post hoc shows that only STPV-C8 anchor is significantly different, and the other relations are not.

Hertz: Young's modulus - significant difference with both statistical tests. Tukey post hoc shows that both Silicon-C16 anchor and Silicon-C8 are significantly different, and the other relations are not.

Compression: area compressibility - significant difference with both statistical tests. Tukey post hoc shows that Silicon-STPV, Silicon-C8 anchor and STPV-C16 anchor are significantly different, whereas Silicon-C16 anchor, STPV-C8 anchor and C8-C16 anchors are not.

Compression: membrane tension - significant difference with both statistical tests. Tukey post hoc shows that Silicon is significantly different from other surfaces, whereas STPV, C8 and C16 anchors are not significantly different. 

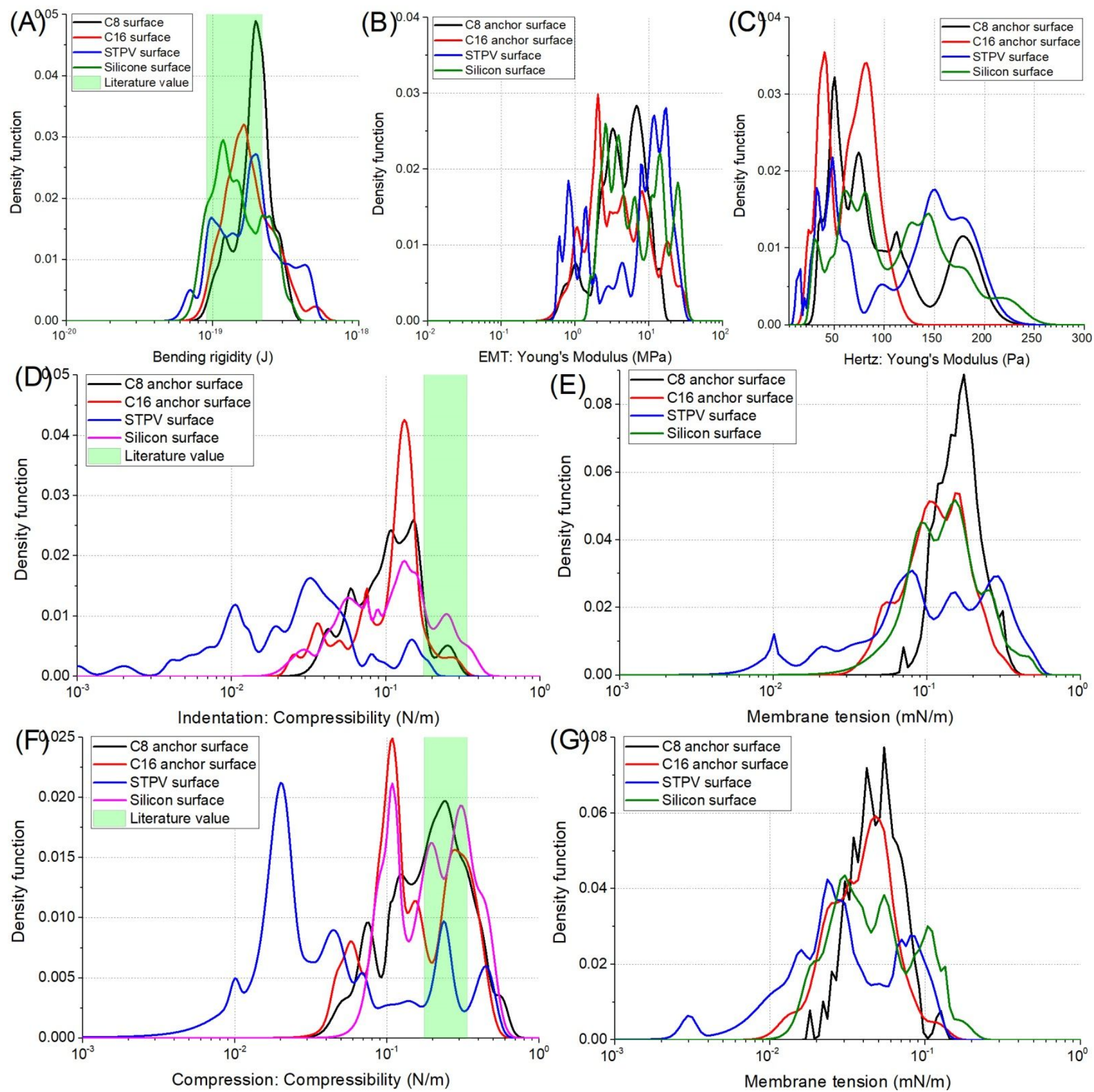

Figure S12. Kernel density representation for parameters obtained from model fitting in function for the different substrates. The results were obtained from the measurements performed using the sphere probe. (A) bending rigidity obtained from the Reissner model. (B) Young's modulus from the Elastic Membrane Theory. (C) Young's modulus from the Hertz model. (D-E) area compressibility $\mathrm{K}_{\mathrm{A}}$ and membrane tension $\sigma$ for the indentation model, respectively. (F-G) area compressibility $\mathrm{K}_{\mathrm{A}}$ and membrane tension $\sigma$ for the compression model, respectively. Reference (green) values were taken from $\underline{33}, \underline{40}, \underline{46}$.

\section{$\underline{\text { Statistical significance }}$}

Bending rigidity - Both statistical tests show no statistically significant difference.

EMT: Young's modulus - significant difference with both statistical tests. Tukey post hoc shows that statistically significant difference occurs between Silicon-C 8 anchor and Silicon-C16 anchor surface pairs.

Hertz: Young's modulus - significant difference with both statistical tests. Tukey post hoc shows that statistically significant difference occurs between Silicon and C16 anchor.

Indentation: area compressibility - significant difference with both statistical tests. Tukey post hoc shows that STPV is significantly different from other surfaces. Silicon, C8 and C16 anchors are not significantly different.

Indentation: membrane tension - Brown-Forsythe ANOVA doesn't report any significant difference, while Kruskal-Wallis does report statistically significant difference between the populations. Detailed information about the relations between the populations are unavailable because Tukey post hoc is calculated only with Brown-Forsythe ANOVA.

Compression: area compressibility - significant difference with both statistical tests. Tukey post hoc shows that STPV-Silicon and STPV-C8 anchor are significantly different. 
Compression: membrane tension - Brown-Forsythe ANOVA reports significant difference between the populations. However, Kruskal-Wallis does not report any statistically significant differences. Tukey post hoc shows that statistically significant difference is only between Silicon-C16 anchor pair of the surface type.

\section{Effect of Biotin}

Below was presented comparison between results obtained on GUVs of the same composition (prepared from the lipid mixtures containing $2 \%$ biotin-DHPE) on two different surfaces - C8 anchor and STPV.
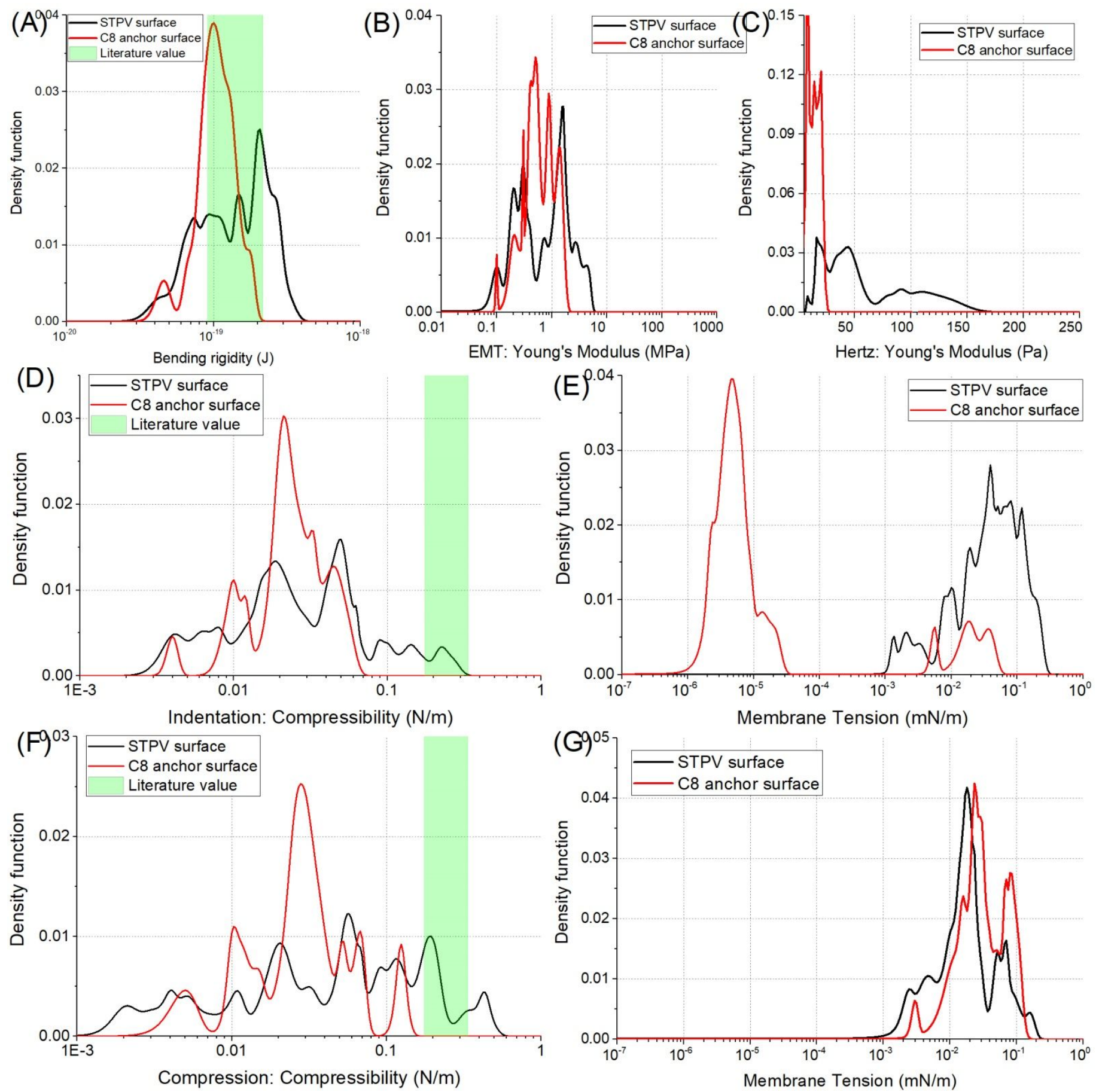

Figure S13. Kernel density representation for parameters obtained from model fitting of GUVs with biotin in function of different substrate. The results were obtained from measurements with cone-shaped tip. (A) bending rigidity obtained from the Reissner model. (B) Young's modulus from the Elastic Membrane Theory. (C) Young's modulus from the Hertz model. (D-E) area compressibility $\mathrm{K}_{\mathrm{A}}$ and membrane tension $\sigma$ for the indentation model, respectively. (F-G) area compressibility $\mathrm{K}_{\mathrm{A}}$ and membrane tension $\sigma$ for the compression model, respectively. Reference (green) values were taken from $\underline{33}, \underline{40}, \underline{46}$.

\section{Statistical significance}

Bending rigidity - both statistical tests show no statistically significant difference.

EMT: Young's modulus - Brown-Forsythe ANOVA reports significant difference between the populations. However, KruskalWallis does not report any statistically significant differences. 
Hertz: Young's modulus - both statistical tests show statistically significant difference.

Indentation: area compressibility - both statistical tests show no statistically significant difference.

Indentation: membrane tension - both statistical tests show statistically significant difference.

Compression: area compressibility - Brown-Forsythe ANOVA reports significant difference between the populations. KruskalWallis does not report any statistically significant differences.

Compression: membrane tension - both statistical tests show statistically significant difference.

Bending rigidity was not statistically significant for the vesicles ( $2 \mathrm{~m} \%$ biotin-DHPE) on the STPV and C 8 anchor surfaces. This shows that the observed difference in bending rigidity on STPV surface was a result of bilayer modification rather than the effect of the surface. Similar observation can be made for the area compressibility - there was no statistically significant difference for the values obtained using both the indentation and compression models (although only with non-parametric test for the latter). In case of the membrane tension population of biotinylated lipids on the C8 anchor surface was similar to POPC lipids on the C8 anchor surface showing that the membrane tension is mostly dependent on surface type.

\section{Effect of the Tip Shape}

Below were presented the rest of the parameters for comparison between the results obtained using either the cone-shaped tip or the sphere probe, for all the investigated surface types. 

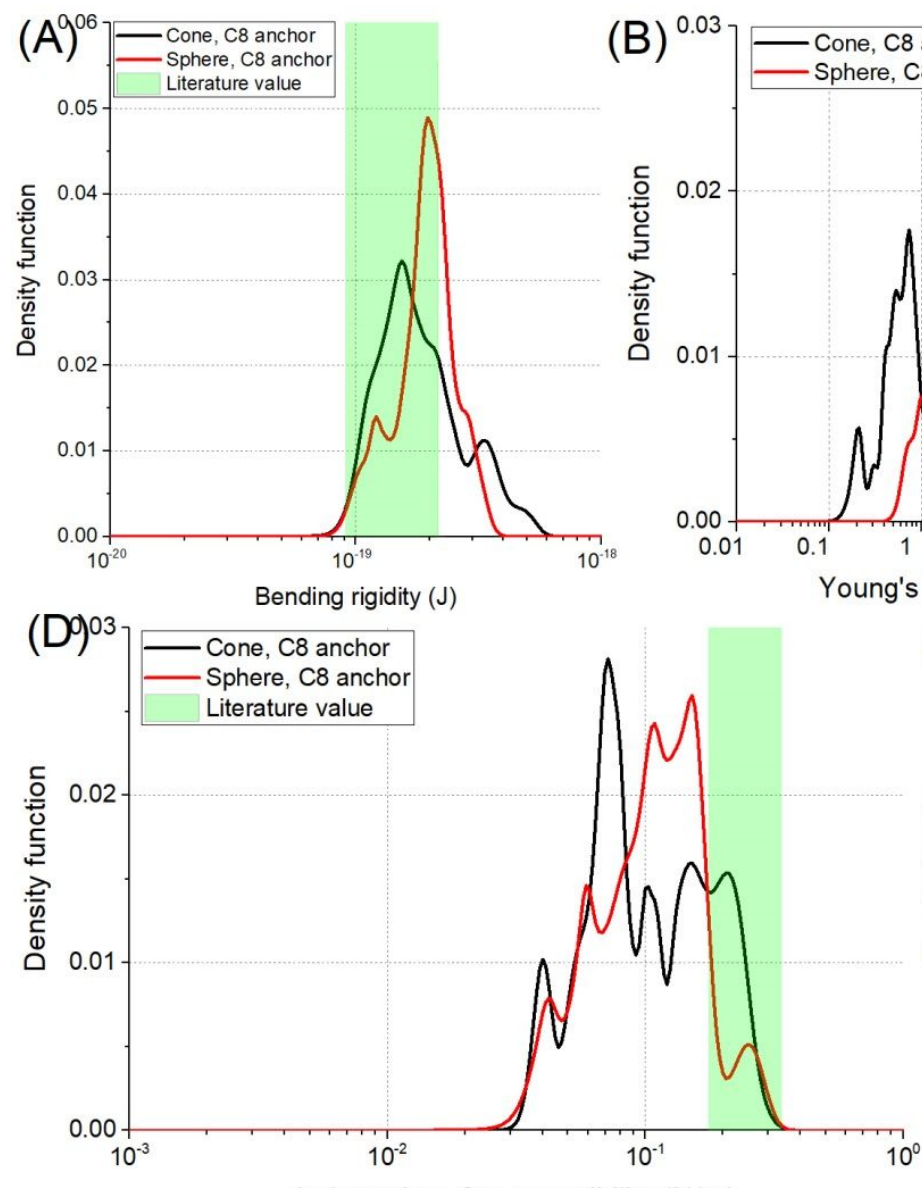

Indentation: Compressibility (N/m)
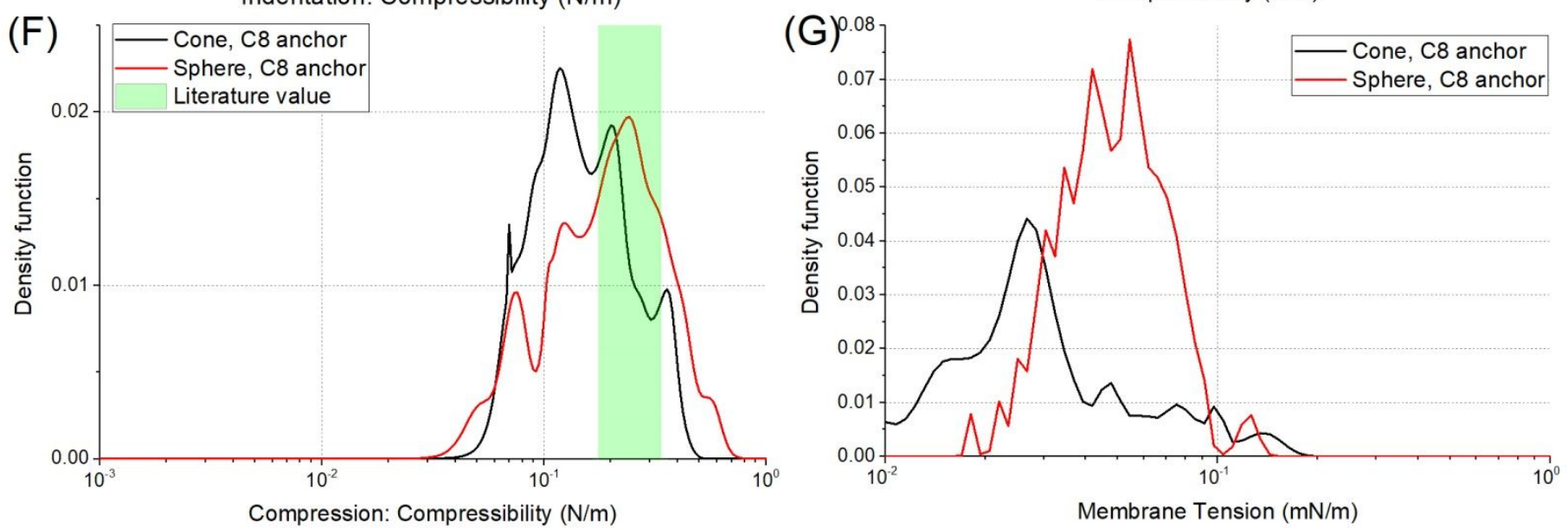

Figure S14. Kernel density representation for parameters obtained from model fitting in function of different tip geometry. The results were obtained from measurements on C8 anchor surface. (A) bending rigidity obtained from the Reissner model. (B) Young's modulus from the Elastic Membrane Theory. (C) Young's modulus from the Hertz model. (D-E) area compressibility $\mathrm{K}_{\mathrm{A}}$ and membrane tension $\sigma$ for the indentation model, respectively. (F-G) area compressibility $\mathrm{K}_{\mathrm{A}}$ and membrane tension $\sigma$ for the compression model, respectively. Reference (green) values were taken from $\underline{33}, \underline{40}, \underline{46}$.

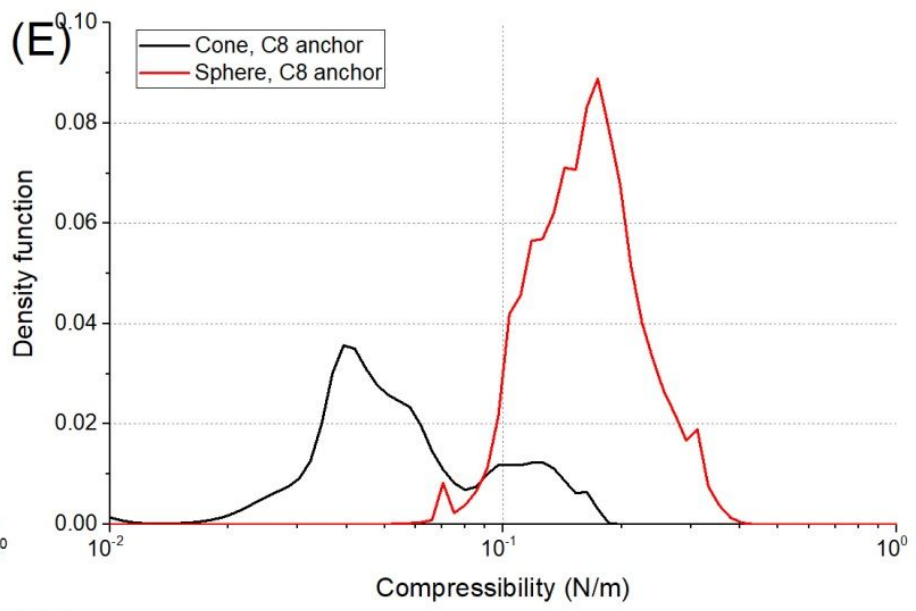



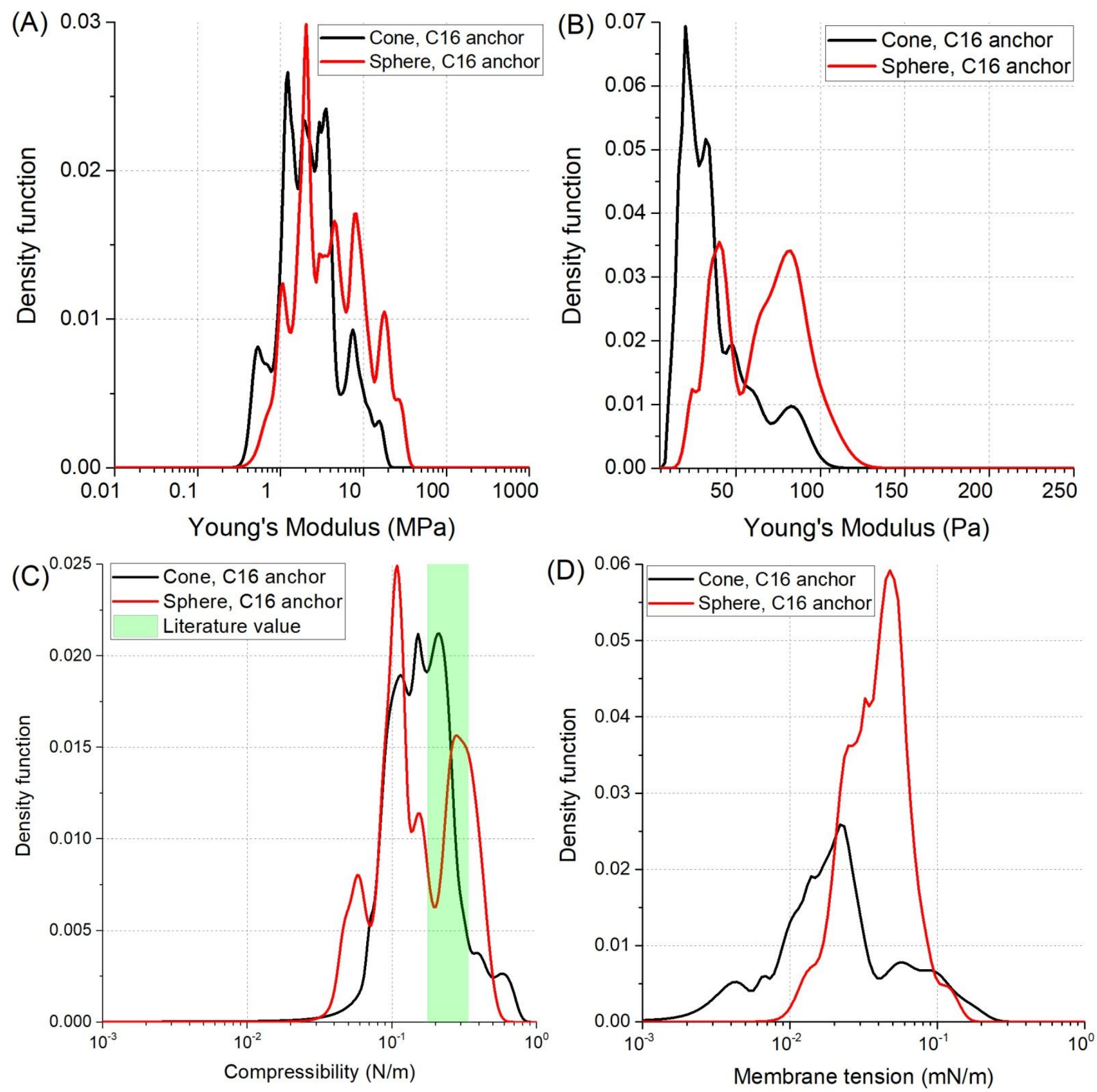

Figure S15. Kernel density representation for parameters obtained from model fitting in function of different tip geometry. The results were obtained from measurements on C16 anchor surface. (A) Young's modulus from the Elastic Membrane Theory. (B) Young's modulus from the Hertz model. (C-D) area compressibility $\mathrm{K}_{\mathrm{A}}$ and membrane tension $\sigma$ for the compression model, respectively. Reference (green) values were taken from $\underline{33}, \underline{40}, \underline{46}$. 

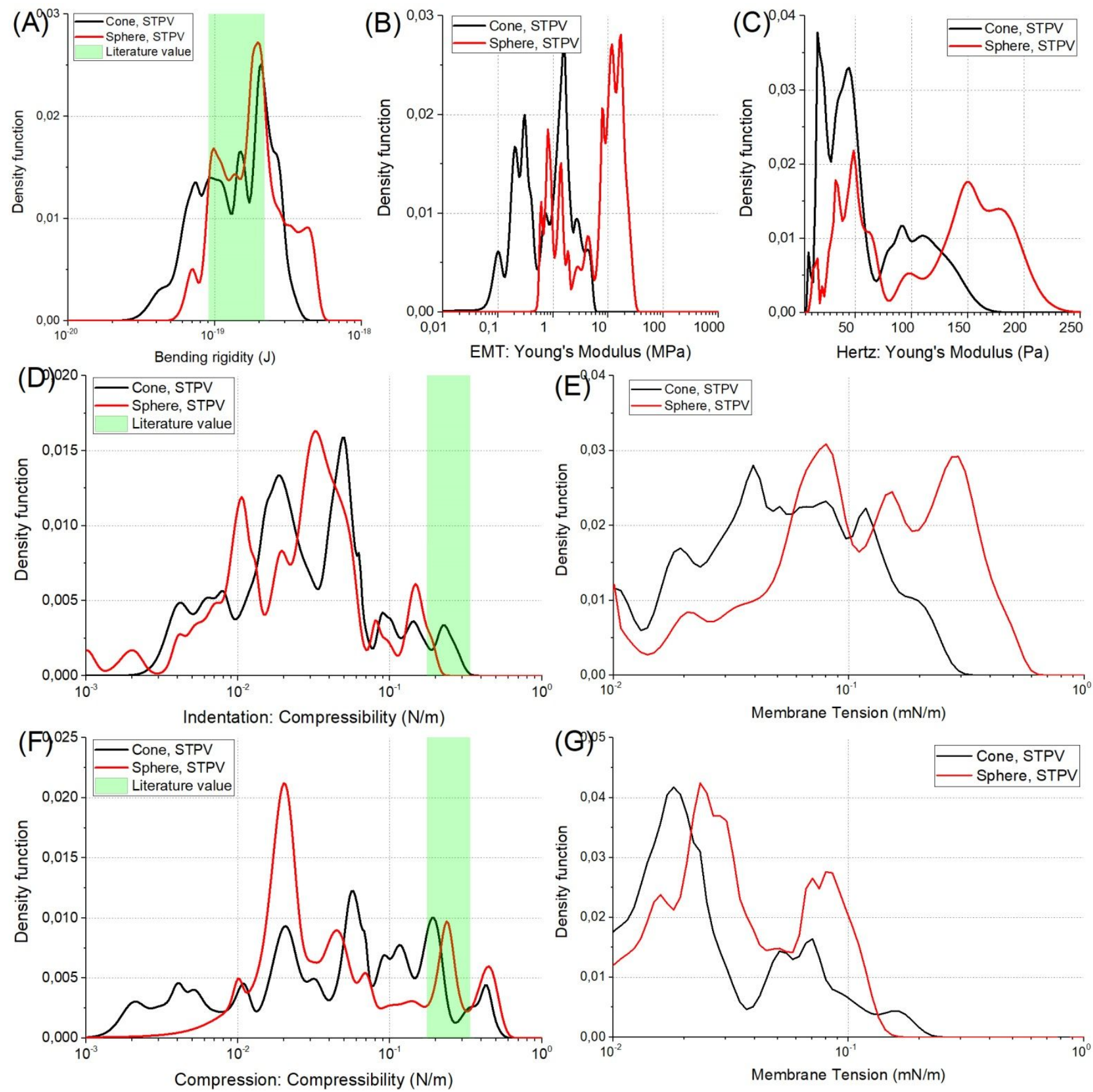

Figure S16. Kernel density representation for parameters obtained from model fitting in function of different tip geometry. The results were obtained from measurements on STPV surface. (A) bending rigidity obtained from the Reissner model. (B) Young's modulus from the Elastic Membrane Theory. (C) Young's modulus from the Hertz model. (D-E) area compressibility $\mathrm{K}_{\mathrm{A}}$ and membrane tension $\sigma$ for the indentation model, respectively. $(\mathrm{F}-\mathrm{G})$ area compressibility $\mathrm{K}_{\mathrm{A}}$ and membrane tension $\sigma$ for the compression model, respectively. Reference (green) values were taken from $\underline{33}, \underline{40}, \underline{46}$. 

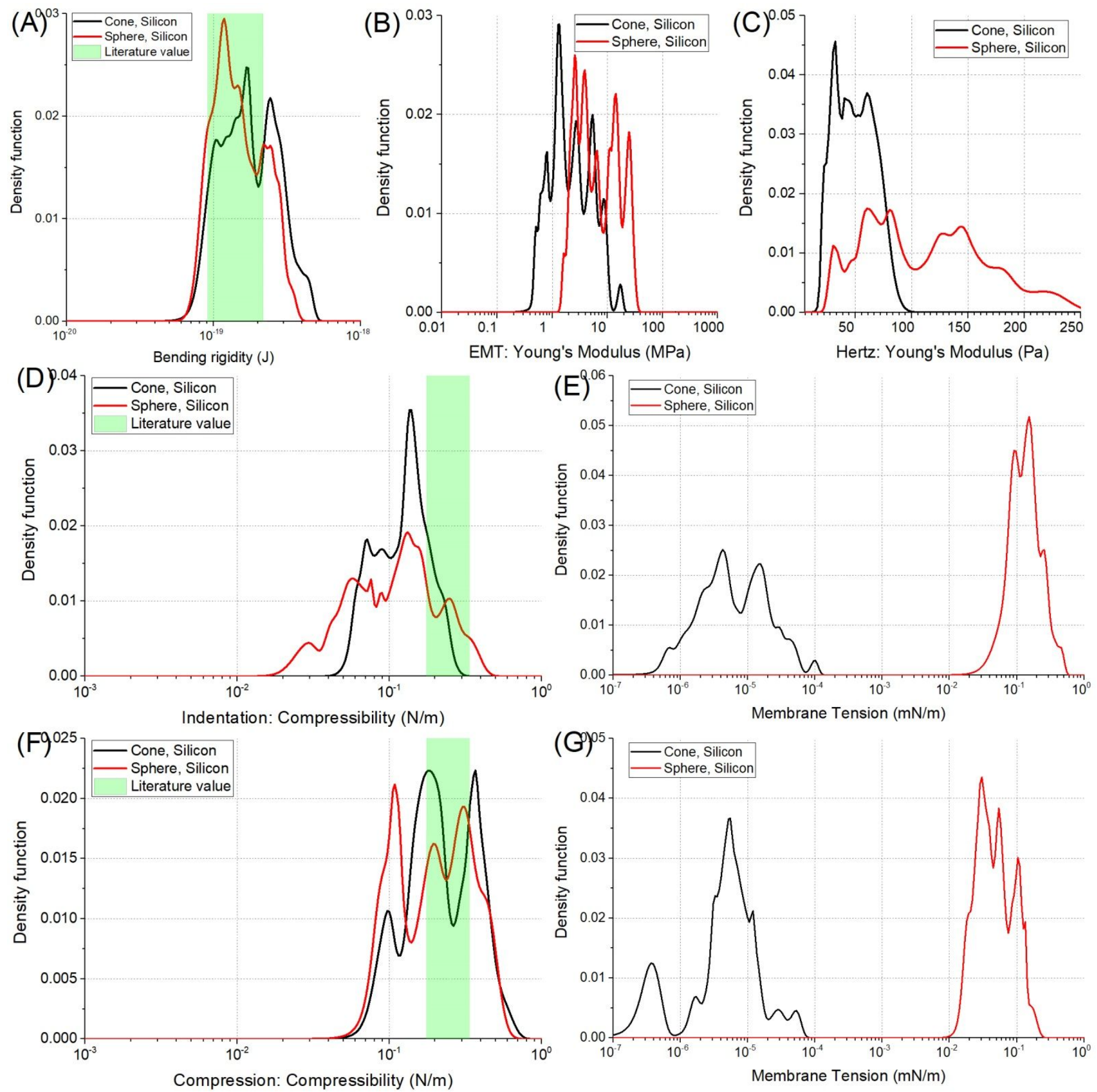

Figure S17. Kernel density representation for parameters obtained from model fitting in function of different tip geometry. The results were obtained from measurements on silicon surface. (A) bending rigidity obtained from the Reissner model. (B) Young's modulus from the Elastic Membrane Theory. (C) Young's modulus from the Hertz model. (D-E) area compressibility $\mathrm{K}_{\mathrm{A}}$ and membrane tension $\sigma$ for the indentation model, respectively. (F-G) area compressibility $\mathrm{K}_{\mathrm{A}}$ and membrane tension $\sigma$ for the compression model, respectively. Reference (green) values were taken from $\underline{33}, 40,46$.

\section{Cholesterol Effect}

Below were presented the rest of the parameters for increasing concentration of cholesterol fraction in lipid membrane measured using the sphere probe, on the $\mathrm{C} 16$ surface. 

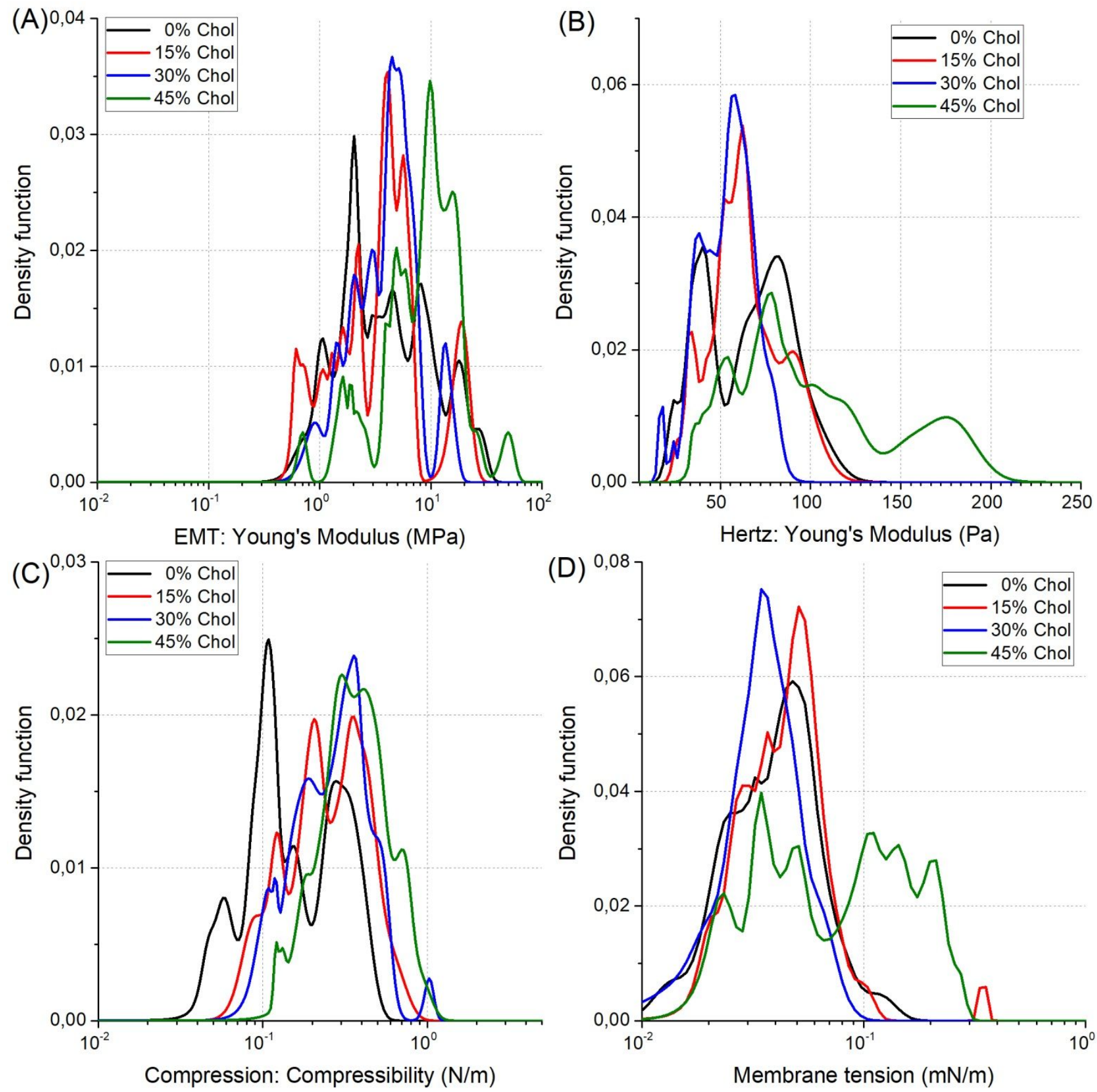

Figure S18. Kernel density representation for parameters obtained from model fitting in function of different cholesterol concentration in POPC bilayer. The results were obtained from measurements on $\mathbf{C 1 6}$ anchor surface and sphere probe. (A) Young's modulus from the Elastic Membrane Theory. (B) Young's modulus from the Hertz model. (C-D) area compressibility $\mathrm{K}_{\mathrm{A}}$ and membrane tension $\sigma$ for the compression model, respectively.

\section{Statistical significance}

EMT: Young's modulus - both tests show statistically significant difference. Tukey post hoc shows that significant difference occurs only between Chol45 and the rest of the populations.

Hertz: Young's modulus - both tests show statistically significant difference. Tukey post hoc shows that significant difference occurs only between Chol45 and the rest of the populations.

Compression: area compressibility - both tests show statistically significant difference. Post hoc Tukey shows that significance occur between the following pairs: Chol45-Chol15, Chol45-Chol0 and Chol30-Chol0. The difference is not high enough to distinguish 'neighboring' populations: Chol30-Chol15, Chol15-Chol0 or Chol45-Chol30.

Compression: membrane tension - both tests show statistically significant difference. Tukey post hoc shows that significant difference occurs only between Chol45 and the rest of the populations. 
Table S2. Median values of the main mechanical parameters for GUVs containing different molar fraction of cholesterol in POPC bilayer. The indicated percentages of cholesterol denote the fraction used in the lipid mixture for preparation of the GUVs.

\begin{tabular}{lllll}
\hline Parameter & Chol 0\% & Chol 15\% & Chol 30\% & Chol 45\% \\
\hline Bending rigidity J & $(1.6 \pm 0.4) \cdot 10^{-19}$ & $(2.1 \pm 0.5) \cdot 10^{-19}$ & $(2.3 \pm 0.3) \cdot 10^{-19}$ & $(2.6 \pm 0.5) \cdot 10^{-19}$ \\
EMT: Young's modulus MPa & $3.1 \pm 1.5$ & $4 \pm 2$ & $4 . \pm 2$ & $9 \pm 5$ \\
Hertz: Young's modulus Pa & $46 \pm 17$ & $57 \pm 11$ & $50 \pm 12$ & $76.0 \pm 22.5$ \\
COM: Compressibility N/m & $0.12 \pm 0.04$ & $0.2 \pm 0.1$ & $0.31 \pm 0.12$ & $0.38 \pm 0.13$ \\
COM: Membrane tension $\mathbf{~ m N / m}$ & $0.042 \pm 0.013$ & $0.048 \pm 0.011$ & $0.037 \pm 0.008$ & $0.07 \pm 0.04$ \\
IND: Compressibility N/m & $0.12 \pm 0.03$ & $0.16 \pm 0.05$ & $0.15 \pm 0.02$ & $0.20 \pm 0.05$ \\
IND: Membrane tension $\mathbf{~ m N / m}$ & $0.12 \pm 0.04$ & $0.12 \pm 0.03$ & $0.106 \pm 0.011$ & $0.22 \pm 0.06$ \\
\hline
\end{tabular}

\section{EMT Young's Modulus correlation with Indentation model's Area Compressibility}

Elastic membrane theory is supposed reflect the influence of shell thickness and structure. In our understanding, Young's modulus obtained from EMT should be related to area compressibility of the membrane. To test that, we investigated possible correlation between the values of Young's modulus obtained from EMT and area compressibility obtained from Indentation model. In Table S3 a summary of the results is presented. The correlation was observed in majority of the repeats. However, the slope of intercept parameters are not consistent within same experimental conditions (lipid type, surface, probe) between different repeats. Since error bars are significant (as presented in figure S19), this difference might result from underestimation of intercept and slope during the linear fitting.

Table S3. Correlation parameters of Young's modulus obtained using EMT and area compressibility from Indentation model.

\begin{tabular}{|c|c|c|c|c|c|c|c|c|c|}
\hline Lipid & Probe & Surface & Repeat & Slope A & $\overline{\Delta \mathbf{A}}$ & Intercept B & $\overline{\Delta B}$ & $R^{\wedge} 2$ & Comment \\
\hline POPC & Cone & $\mathrm{C} 8$ & 1 & 0,008 & 0,001 & 0,053 & 0,014 & $79,29 \%$ & \\
\hline POPC & Cone & $\mathrm{C} 8$ & 2 & 0,014 & 0,001 & 0,028 & 0,006 & $96,76 \%$ & \\
\hline POPC & Cone & $\mathrm{C} 8$ & 3 & 0,020 & 0,004 & 0,044 & 0,011 & $76,62 \%$ & \\
\hline POPC & Sphere & $\mathrm{C} 8$ & 1 & 0,010 & 0,003 & 0,051 & 0,016 & $63,40 \%$ & \\
\hline POPC & Sphere & $\mathrm{C} 8$ & 2 & 0,015 & 0,004 & 0,037 & 0,030 & $69,40 \%$ & \\
\hline POPC & Sphere & $\mathrm{C} 8$ & 3 & 0,017 & 0,002 & 0,012 & 0,013 & $89,71 \%$ & \\
\hline POPC & Cone & $\mathrm{C} 16$ & 1 & 0,024 & 0,003 & 0,009 & 0,014 & $74,48 \%$ & \\
\hline POPC & Cone & $\mathrm{C} 16$ & 2 & 0,015 & 0,001 & 0,037 & 0,004 & $97,44 \%$ & \\
\hline POPC & Cone & $\mathrm{C} 16$ & 3 & 0,074 & 0,011 & $-0,038$ & 0,018 & $88,00 \%$ & \\
\hline POPC & Sphere & C16 & 1 & 0,008 & 0,002 & 0,047 & 0,006 & $61,19 \%$ & \\
\hline POPC & Sphere & C16 & 2 & 0,015 & 0,003 & $-0,007$ & 0,014 & $79,86 \%$ & \\
\hline POPC & Sphere & $\mathrm{C} 16$ & 3 & 0,044 & 0,017 & 0,005 & 0,040 & $38,40 \%$ & no correlation \\
\hline POPC & Cone & Silicon & 1 & 0,007 & 0,001 & 0,068 & 0,006 & $78,93 \%$ & \\
\hline POPC & Cone & Silicon & 2 & 0,034 & 0,016 & 0,054 & 0,025 & $60,87 \%$ & \\
\hline POPC & Cone & Silicon & 3 & 0,024 & 0,004 & 0,040 & 0,017 & $82,20 \%$ & \\
\hline POPC & Sphere & Silicon & 1 & 0,004 & 0,001 & 0,068 & 0,011 & $81,28 \%$ & \\
\hline POPC & Sphere & Silicon & 2 & 0,012 & 0,001 & 0,020 & 0,018 & $87,47 \%$ & \\
\hline POPC & Sphere & Silicon & 3 & 0,014 & 0,002 & 0,041 & 0,013 & $78,27 \%$ & \\
\hline POPC & Cone & STPV & 1 & 0,015 & 0,002 & 0,004 & 0,001 & $89,46 \%$ & Fig S18.C \\
\hline POPC & Cone & STPV & 2 & 0,012 & 0,000 & 0,012 & 0,003 & $98,92 \%$ & \\
\hline POPC & Cone & STPV & 3 & 0,020 & 0,002 & 0,030 & 0,009 & $89,78 \%$ & \\
\hline POPC & Sphere & STPV & 1 & 0,006 & 0,001 & 0,010 & 0,006 & $90,96 \%$ & \\
\hline POPC & Sphere & STPV & 2 & 0,002 & 0,001 & 0,012 & 0,010 & $37,33 \%$ & no correlation \\
\hline POPC & Sphere & STPV & 3 & 0,004 & 0,003 & 0,007 & 0,003 & $13,56 \%$ & no correlation \\
\hline POPC+Bio & Cone & Silicon & 1 & 0,033 & 0,003 & 0,002 & 0,001 & $93,38 \%$ & Fig S18.A \\
\hline Chol15\% & Sphere & $\mathrm{C} 16$ & 1 & 0,050 & 0,010 & $-0,138$ & 0,050 & $72,26 \%$ & \\
\hline Chol15\% & Sphere & $\mathrm{C} 16$ & 2 & 0,013 & 0,004 & 0,152 & 0,048 & $57,95 \%$ & $\begin{array}{l}\text { possible } \\
\text { correlation }\end{array}$ \\
\hline Chol30\% & Sphere & $\mathrm{C} 16$ & 1 & 0,024 & 0,005 & $-0,027$ & 0,009 & $79,94 \%$ & Fig S18.B \\
\hline Chol30\% & Sphere & $\mathrm{C} 16$ & 2 & 0,012 & 0,008 & 0,080 & 0,043 & $20,75 \%$ & no correlation \\
\hline Chol45\% & Sphere & C16 & 1 & 0,008 & 0,002 & 0,085 & 0,030 & $47,35 \%$ & $\begin{array}{l}\text { possible } \\
\text { correlation }\end{array}$ \\
\hline Chol45\% & Sphere & $\mathrm{C} 16$ & 2 & 0,094 & 0,011 & $-0,233$ & 0,064 & $95,03 \%$ & \\
\hline
\end{tabular}



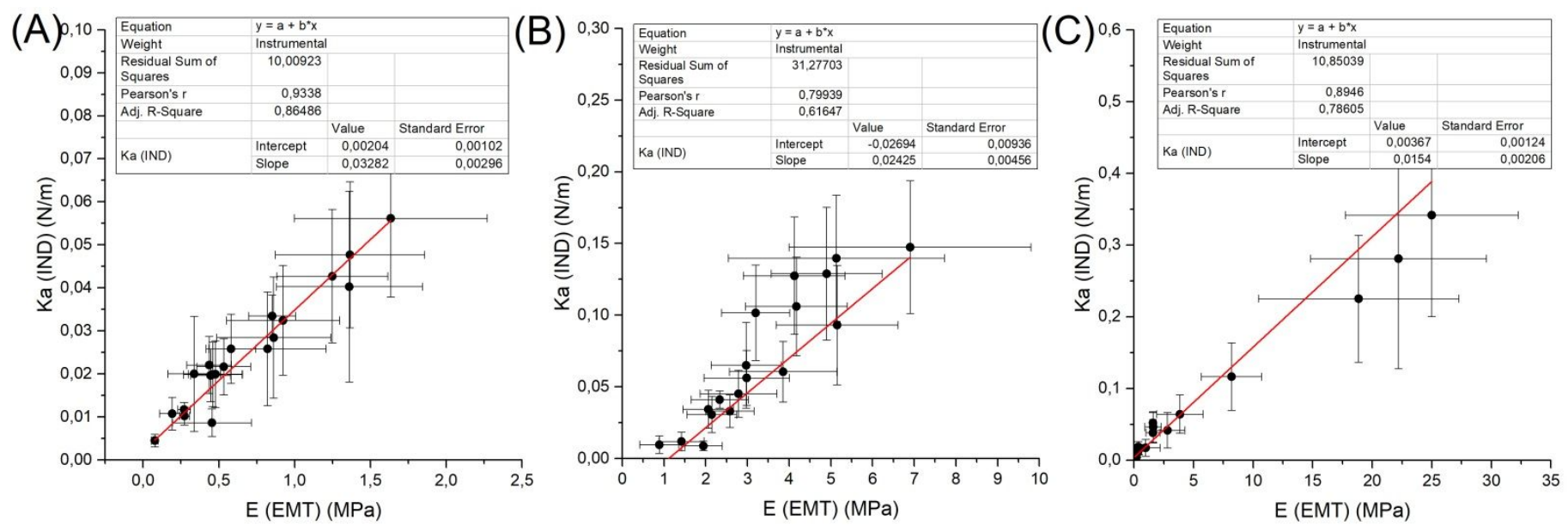

Figure S19. Randomly selected correlation plots of Young's modulus obtained using EMT and area compressibility obtained using indentation model. In Table S3 it is marked with repeats are presented in A-C.

\section{Raw Data Force curves and GUVs diameter population}

\#2 Repeat, C8 surface, cone-shaped tip

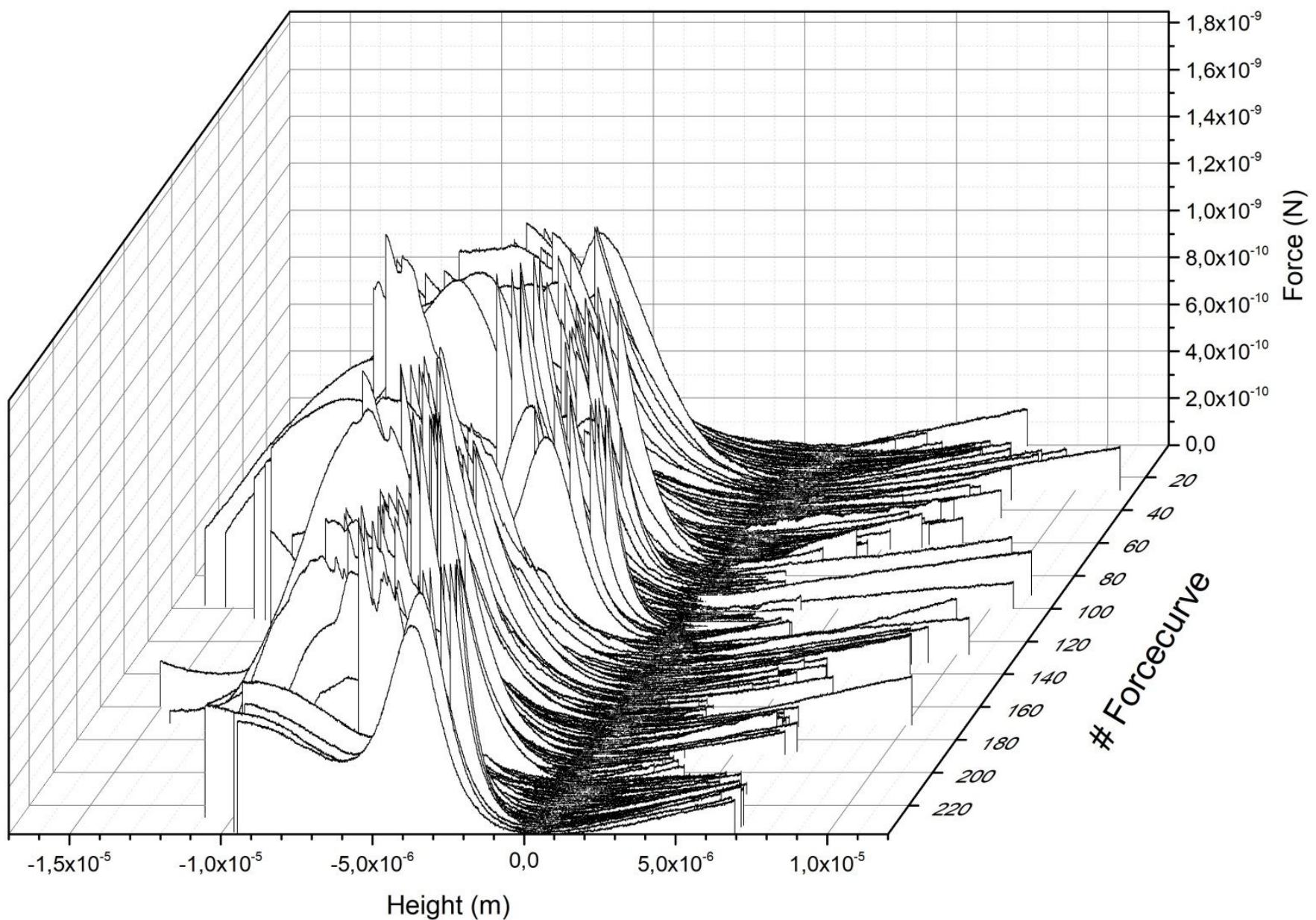

Figure S20. Raw force curves from the second repeat for a measurement on the C8 anchor surface using a cone-shaped tip. In total, 237 force curves are presented. Number of vesicles analysed in this repeat were equal to 15 . From 14 up to 20 force curves were recorded from a single vesicle. 


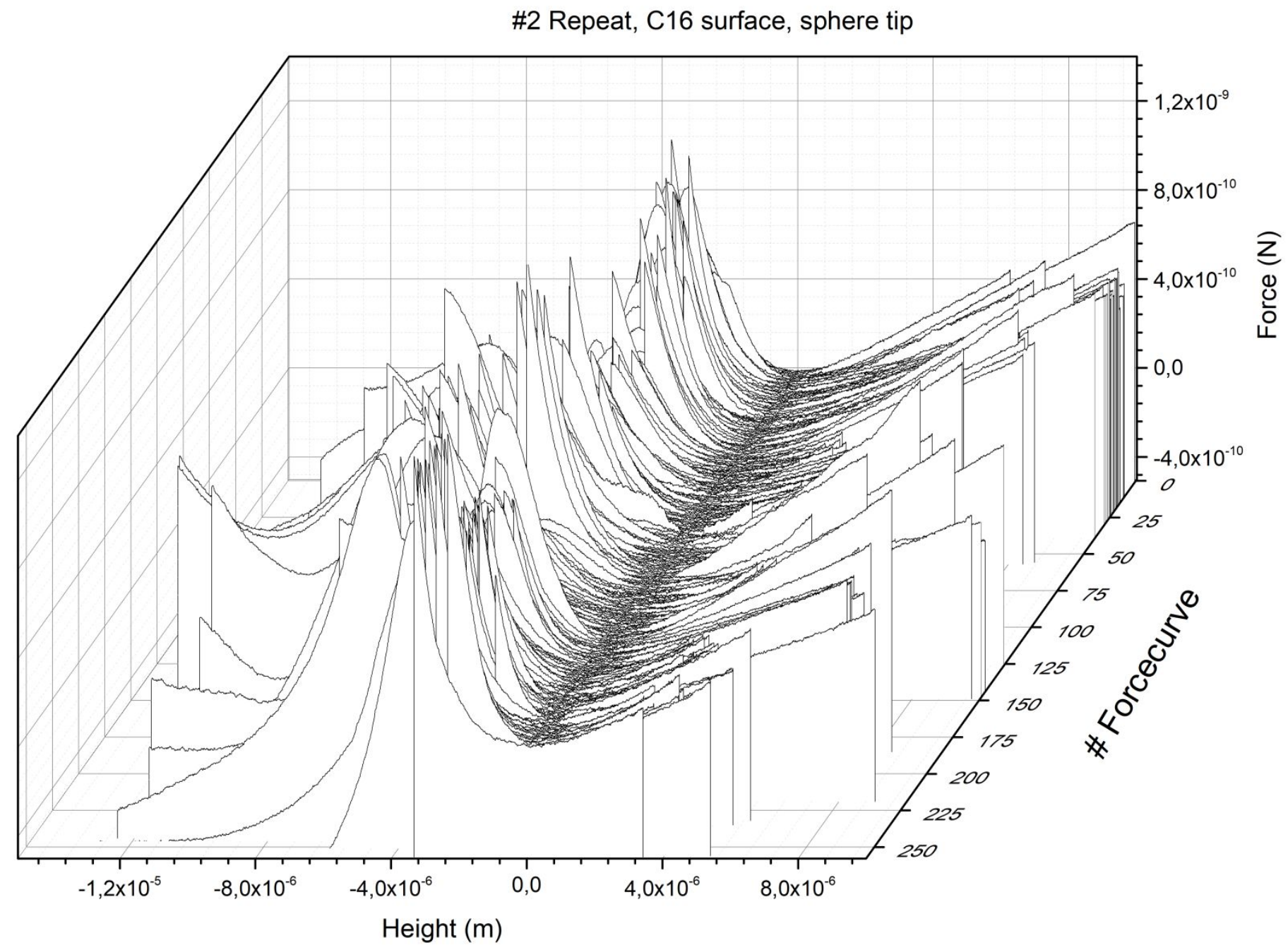

Figure S21. Raw force curves from the second repeat for a measurement on the C16 anchor surface using a sphere tip. In total, 256 force curves are presented. Number of vesicles analysed in this repeat were equal to 15 . From 14 up to 20 force curves were recorded from a single vesicle.

Experimental data is available from the corresponding author upon reasonable request. 


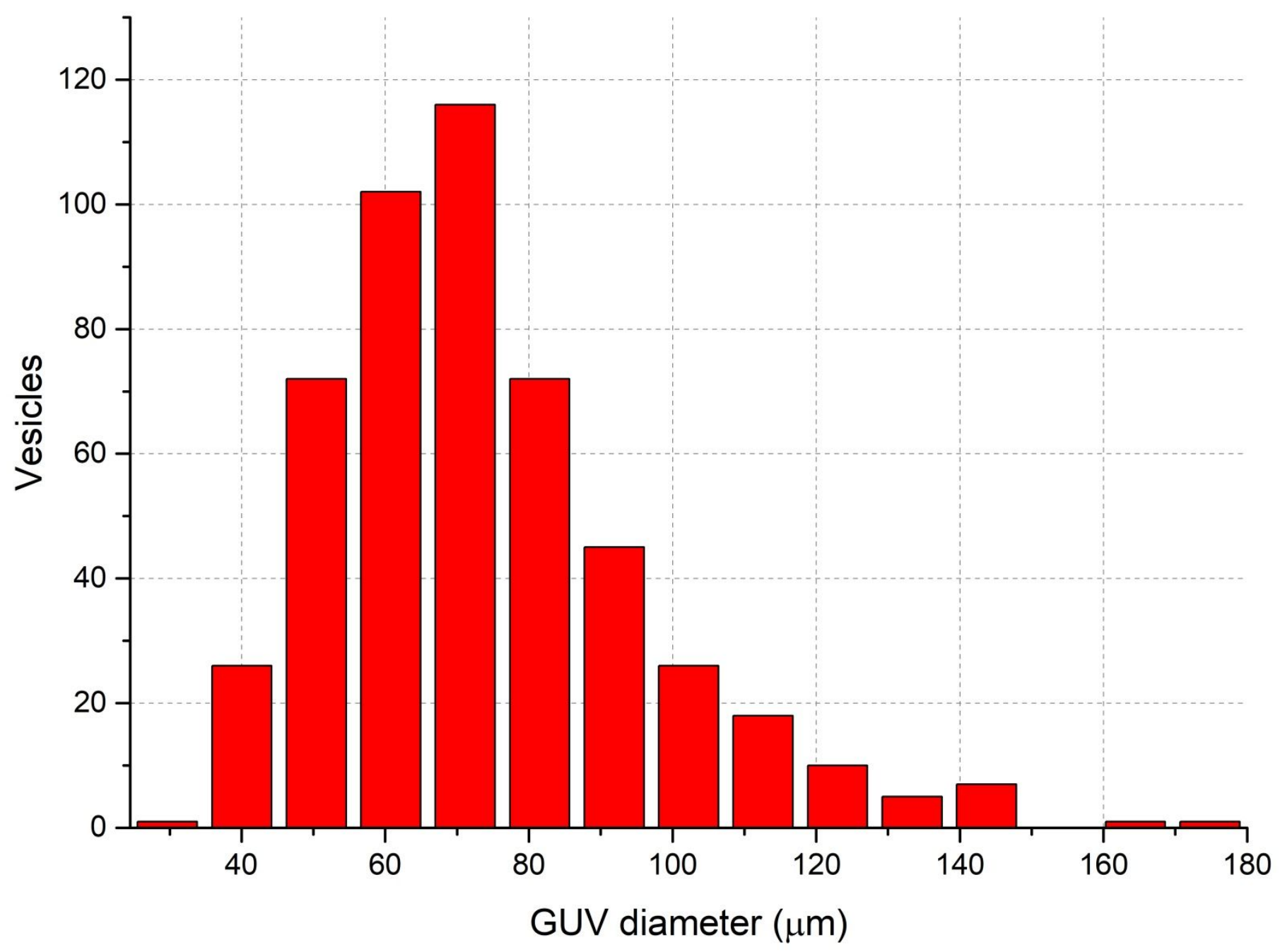

Figure S22. Histogram of GUVs diameters used for mechanical properties determination. Histogram was done on all vesicles that were used throughout all measurements. 